\title{
The Alexander module of a trigonal curve
}

\author{
Alex Degtyarev
}

\begin{abstract}
We describe the Alexander modules and Alexander polynomials (both over $\mathbb{Q}$ and over finite fields $\mathbb{F}_{p}$ ) of generalized trigonal curves. The rational case is completely resolved; in the case of characteristic $p>0$, a few points remain open. The results obtained apply as well to plane curves with deep singularities.
\end{abstract}

\section{Introduction}

\subsection{Motivation}

This paper continues the systematic study of the fundamental groups of (generalized) trigonal curves that was started in [6]. (By a common abuse of language, when speaking about the fundamental group of an embedded curve, one means the group of the complement of the curve; see Subsection 3.4 for the precise description of the groups to be studied.) The principal motivation for this research is the belief that there should be strong restrictions on the complexity of these groups, far beyond the obvious fact that they admit presentations with at most three generators. Thus, only about a dozen distinct groups appear as the fundamental groups of irreducible plane sextics with a triple point (see [10] and references therein), which are a special class of generalized trigonal curves. (Remarkably, the commutants of most finite groups obtained in this way are of the form $S L(2, \mathbb{k})$, where $\mathbb{k}$ is a finite field.) These restrictions are due to the fact that the monodromy group of a trigonal curve is a genus zero subgroup of the modular group, see Subsection 3.2 and Theorem 3.2; hence, it is sufficiently 'large', resulting in a sufficiently small fundamental group. At present, it is not quite clear how or even in what terms such fundamental groups can be characterized; as a first step, we make an attempt to describe their metabelian invariants.

Another special feature of trigonal curves is the fact that, in this case, the relation between the fundamental group and the geometry of a curve is 'twosided', as all curves with 'at least' a certain fundamental group are essentially

Mathematics Subject Classification (2010): Primary 14H30; Secondary 14H45, 14H50, 20F36. Keywords: Trigonal curve, fundamental group, Alexander module, Alexander polynomial, Burau representation, modular group. 
induced from some universal curve with this property, see Speculation 1.2.1 and a number of examples in [6]. For example, Theorem 1.2.5 in [6] characterizes the so-called curves of torus type in terms of their Alexander polynomials; remarkably, a very similar assertion holds for irreducible plane sextics, see [9]. An essential intermediate statement concerning the universal curves is recalled as Theorem 3.2.

A generalized trigonal curve in the Hirzebruch surface $\Sigma_{1}$ (the plane blown up at one point) can be regarded as a curve in the plane $\mathbb{P}^{2}=\Sigma_{1} / E$, where $E$ is the exceptional section, and as such it has a distinguished singular point of multiplicity (degree - 3), see Subsection 3.5. Thus, the study of trigonal curves sheds light on the classical problem about the fundamental group of a plane curve. (It is this construction that motivated my original interest in trigonal curves.) As an example, the passage to the trigonal model, combined with the techniques of dessins d'enfants described below, lets one compute the fundamental groups of all irreducible sextics with a singular point of multiplicity at least three, see [10], whereas the groups of a number of sextics with only double singular points are still unknown. It is worth mentioning that there is a mysterious similarity, although not quite literal coincidence, between the properties of plane sextics and those of trigonal curves (see [6] for a more detailed discussion); it must be due to the similarity between $K 3$ - and elliptic surfaces.

The principal tool used in this paper is the correspondence between trigonal curves in Hirzebruch surfaces, genus zero subgroups of the modular group, and a certain class of planar bipartite ribbon graphs (essentially, Grothendieck's dessins d'enfants for the modular $j$-invariant), see, e.g., [2], [3], [6], [11], and[14]. As a byproduct, we obtain some information on the sparseness of the image of the Burau representation of the braid group $\mathbb{B}_{3}$, see Remark 1.6 on the 'Burau congruence subgroups', although no attempt to formalize these results has been made.

\subsection{The subject}

In [6], we gave a complete classification of the dihedral quotients of the fundamental group of a generalized trigonal curve. Here, we deal with the ultimate metabelian invariants of a curve, viz. its so-called Alexander module and Alexander polynomial. In the context of algebraic curves, this concept appeared essentially in [23]; it was later developed in [15], [16], [17] and [18], and it has been a subject of intensive research since then, see the recent surveys [19] and [21] for further references.

For an irreducible generalized trigonal curve $C$ in the Hirzebruch surface $\Sigma_{d}$ (see Section 3), the Alexander module $\mathrm{A}_{C}$ can be defined as the homology group $H_{1}(X)$ of the maximal cyclic covering $X \rightarrow \Sigma_{d}$ ramified at $C$ and the exceptional section $E$, see Subsections 2.6 and 3.4 for details. The deck translation automorphism of the covering induces an action on $\mathrm{A}_{C}$, turning it into a module over the ring $\Lambda:=\mathbb{Z}\left[t, t^{-1}\right]$ of Laurent polynomials. This module describes the fundamental group of the curve modulo its second commutant. Classically, one tensors $\mathrm{A}_{C}$ by $\mathbb{Q}$ to get a torsion module over the principal ideal domain $\Lambda \otimes \mathbb{Q}$; the order $\Delta_{C}$ of $\mathrm{A}_{C} \otimes \mathbb{Q}$ is called the Alexander polynomial of $C$. To capture the integral torsion of $\mathrm{A}_{C}$, we will also consider the product $\mathrm{A}_{C} \otimes \mathbb{F}_{p}$ for a prime $p$; the order $\Delta_{C, p} \in \Lambda \otimes \mathbb{F}_{p}$ of this product is called the $(\bmod p)$-Alexander polynomial. 
(A similar approach was used in [16], where some $(\bmod p)$-Alexander polynomials were computed.)

As in the knot theory, the Alexander polynomial is a purely algebraic invariant of the fundamental group of the curve, but it is usually much easier to compute directly. The classical rational polynomial $\Delta_{C}(t)$ can be computed by means of Hodge theory, in terms of the superabundance of certain linear systems related to the singularities of the curve, see [7], [13], [16], and [20]. (Although most results are stated for plane curves, they can easily be adapted to curves in any surface.) Moreover, there are many so-called divisibility theorems bounding the Alexander polynomial in terms of the degree of the curve and/or its singularities. Some of these theorems, e.g., [15], [16], are of a purely topological nature and apply as well to pseudo-holomorphic curves and $(\bmod p)$-Alexander polynomials. Others, e.g., [8], rely on vanishing theorems in algebraic geometry; these give better estimates, but work only for algebraic curves and rational Alexander polynomial. All these statements are in sharp contrast with the principal results of this paper, as we show that, for each $p$, the $(\bmod p)$-Alexander polynomial of a trigonal curve may take only finitely many values, no matter what the singularities are. The particular case $p=0$, see Theorem 1.2 , can be translated into a certain restriction on the complexity of the singularities of a trigonal curve and their mutual positions: the superabundance of some linear systems cannot be too large.

\subsection{Principal results}

Throughout the paper, we assume that $p$ is a prime or zero and let $\mathbb{k}_{0}=\mathbb{Q}$ and $\mathbb{k}_{p}=\mathbb{F}_{p}$ for $p>0$. (When $p$ is fixed, we abbreviate $\mathbb{k}_{p}$ to $\mathbb{k}_{\mathrm{k}}$.) For an element $\xi$ algebraic over $\mathbb{k}$, we denote by $\varkappa_{\xi} \in \mathbb{k}[t]$ its minimal polynomial and, if $\xi$ is understood, we let $\mathbb{K}=\mathbb{k}(\xi)=(\Lambda \otimes \mathbb{k}) / \varkappa_{\xi}$. The cyclotomic polynomial (over $\mathbb{Q}$ ) of order $n$ is denoted by $\Phi_{n}$.

As this paper is just a first step towards the understanding of the Alexander module, we choose to work over a field and consider the specializations $\mathrm{A}_{C}(\xi):=$ $\left(\mathrm{A}_{C} \otimes \mathbb{k}\right) / \varkappa_{\xi}$, see Subsection 2.7, thus reducing to $r=1$ the higher torsion summands of the form $\mathbb{Z}_{p^{r}}$ or $(\Lambda \otimes \mathbb{k}) / \varkappa_{\xi}^{r}, r>1$, which may and do appear when $p>0$. In other words, we are trying to enumerate the possible roots $\xi$ of the Alexander polynomial $\Delta_{C, p}$ or, equivalently, its irreducible factors, which are of the form $\varkappa_{\xi}$. Note that $\mathrm{A}_{C}(\xi)$ is a vector space over $\mathbb{K}$, and therefore we can speak about its dimension rather than rank.

Convention 1.1. Since $\Delta_{C, p}$ is defined over $\mathbb{k}_{p}$ itself, the set of its roots is Galois invariant. For this reason, in most statements we refer to the minimal polynomials $\varkappa_{\xi} \in \mathbb{k}_{p}[t]$ rather than to particular roots $\xi \in \mathbb{K}_{p}$. With $\xi$ or $\varkappa_{\xi}$ understood, we fix the notation $N$ for the multiplicative order ord $(-\xi)$. Certainly, $N$ is determined by $p$ and $\varkappa_{\xi}$; however, in view of the importance of this parameter, we will speak about triples $\left(p, N, \varkappa_{\xi}\right)$ rather than just pairs $\left(p, \varkappa_{\xi}\right)$ (or even singletons $\varkappa_{\xi}$, which formally retain the information abou $\mathbb{k}_{p}$ as their coefficient field). It is worth mentioning that each pair $(p, N), N \geqslant 1$, corresponds to but finitely many minimal polynomials $\varkappa_{\xi}$, viz. the irreducible divisors (over $\mathbb{k}_{p}$ ) of $\Phi_{N}(-t)$, and in some statements it is $(p, N)$ that is fixed or discussed, whereas $\varkappa_{\xi}$ is allowed to vary. 
TABLE 1. Exceptional factors of $\Delta(N>10)$

\begin{tabular}{rrlc}
\multicolumn{1}{c}{$p$} & $\mathrm{~N}$ & \multicolumn{1}{c}{ Factors $\varkappa_{\xi} \in \mathbb{F}_{p}[t]$ of $\Delta$} & $\bar{G} \subset \Gamma$ \\
\hline 2 & $* 15$ & $t^{4}+t+1, t^{4}+t^{3}+1$ & $\left(17 ; 1,2 ; 1^{2} 15^{1}\right)$ \\
5 & 12 & $t^{2}+2 t+4, t^{2}+3 t+4$ & $\left(52 ; 0,4 ; 1^{4} 12^{4}\right)$ \\
13 & $* 12$ & $t+2, t+6, t+7, t+11$ & $\left(14 ; 0,2 ; 1^{2} 12^{1}\right)$ \\
19 & 18 & $t+2, t+3, t+10, t+13, t+14, t+15$ & $\left(40 ; 2,4 ; 1^{2} 2^{1} 18^{2}\right)$
\end{tabular}

The principal results of the paper are summarized in the next four statements. We resolve completely the case $p=0$, while for $p>0$ a certain range still remains open. Conjecturally, the Alexander polynomial of a non-isotrivial trigonal curve can take finitely many values, and all irreducible factors are indeed listed in the paper (with Table 3 in Example 5.12 taken into account). Note that, unlike a number of known divisibility theorems ( $c f .[15],[16]$, and [8]), the bounds below are universal, as we do not make any assumptions about the singularities of the curve or its degree.

Theorem 1.2. The Alexander polynomial $\Delta_{C}$ of an irreducible non-isotrivial generalized trigonal curve $C$ can take only the following four values: $\Phi_{6}, \Phi_{6}^{2}, \Phi_{10}$, and $\Phi_{10}^{2}$. All four values can be realized by genuine trigonal curves.

Theorem 1.3. Let $p>0$, and assume that the $(\bmod p)$-Alexander polynomial $\Delta_{C, p}$ of a non-isotrivial generalized trigonal curve $C$ has a root $\xi \in \mathbb{K} \supset \mathbb{k}_{p}$. Then, with the exception of the fourteen triples $\left(p, N, \varkappa_{\xi}\right)$ listed in Table 1 , one has $1 \leqslant N \leqslant 10$. If $C$ is irreducible and $N \neq 3$ or 5 , one has $\operatorname{dim}_{\mathbb{K}} \mathrm{A}_{C}(\xi)=1$.

Addendum 1.4. In the setting of Theorem 1.3, assume in addition that $N \leqslant 5$ and $C$ is irreducible. Then the pair $(p, N)$ and the dimension $r:=\operatorname{dim}_{\mathbb{K}} \mathrm{A}_{C}(\xi)$ take the following values:

1. $(p, N)=(3,4)$ and $r=1$;

2. $(p, N)=(3,1)$ or $(p, 3), p \neq 3$, with $r \leqslant 2$;

3. $(p, N)=(5,1)$ or $(p, 5), p \neq 5$, with $r \leqslant 2$;

4. $(p, N)=(7,1)$ and $r=1$.

All four possibilities for $(p, N)$ (and all possibilities for $r$ ) are realized by genuine trigonal curves, and for such curves they are mutually exclusive.

Addendum 1.5. For each pair $(p, N)$ as in Table 1 , at most one of the factors $\varkappa_{\xi}$ listed can appear in the Alexander polynomial of any given curve. The six triples $\left(p, N, \varkappa_{\xi}\right)$ marked with $a^{*}$ in the table do appear in the Alexander polynomials of genuine trigonal curves; the other eight do not.

Theorem 1.2 is proved in Subsection 6.7. Theorem 1.3 and Addendum 1.5 are proved in Subsection 5.4, and Addendum 1.4 merely summarizes the detailed description of the modules $\mathrm{A}_{C} / \Phi_{N}(-t), N \leqslant 5$, given in Subsections 6.2-6.4. 
In Table 1, the last column gives a description of the projection to the modular group $\Gamma:=P S L(2, \mathbb{Z})$ of the corresponding universal subgroup, see Definition 2.14. Listed are the index $[\Gamma: \bar{G}]$, the numbers $c_{2}, c_{3}$ of the conjugacy classes of elements of order 2 and 3 , respectively, and the set of cusp widths in the partition notation, see [5]. These data do not determine the subgroup completely, but drawing large diagrams does not seem practical here. Note that, in each case marked with $\mathrm{a}^{*}$, the universal subgroup $\bar{G}^{\prime}$ corresponding to genuine trigonal curves is smaller than the one listed: one has $\left[\bar{G}: \bar{G}^{\prime}\right]=3$. Each time, the skeleton of $\bar{G}$, see Subsection 2.4, has one monovalent $\bullet$-vertex and one monogonal region with the type specification nontrivial modulo 6 , see Subsection 2.5, and the skeleton of $\bar{G}^{\prime}$ is the triple cyclic covering ramified at these vertices and regions.

\subsection{Ramifications and speculations}

The assumption that the trigonal curve in question be irreducible is not very important. Lifting this requirement would result in a few extra factors with $N=$ 1,2 , or 4 ; they are controlled by congruence subgroups and thus can easily be enumerated, see Subsections 6.3 and 6.1. (The case $N=1$ is known, see [6].)

As an extra remark, note that, for genuine trigonal curves, each triple $\left(p, N, \varkappa_{\xi}\right)$ among those listed appears in the Alexander polynomial 'in a unique way', in the sense that, up to Nagata equivalence, each curve $C$ with $\varkappa_{\xi} \mid \Delta_{C, p}$ is induced from a certain universal curve with this property, see Subsection 3.1 for the definitions. This statement follows from the uniqueness of the corresponding universal subgroups (found in the computation) and Theorem 3.2.

All four statements apply equally well to plane curves with a singular point of multiplicity deg -3 (as they can be regarded as generalized trigonal curves in the Hirzebruch surface $\Sigma_{1}$, see Subsection 3.5), provided that the trigonal model of the curve is not isotrivial. The relatively simple case of irreducible isotrivial curves is discussed in Subsection 4.7; the degrees of the Alexander polynomials of such curves are not universally bounded.

The parabolic case $N=6$ is treated in Section 7; we do not mention it here as it does not seem to lead to nontrivial conventional Alexander polynomials. (In fact, we mainly study the so-called extended Alexander polynomials, which depend on the monodromy group of the curve rather than on its fundamental group only, see Definition 2.12 and Remark 2.13.) The range $7 \leqslant N \leqslant 10$ remains open. A few examples are found in Table 3 in Example 5.12. I conjecture ${ }^{1}$ that Tables 1 and 3 do exhaust all possibilities with $N \geqslant 7$. Among other consequences, this conjecture would imply that, as an abelian group, $\mathrm{A}_{C}$ has $p$-torsion for finitely many primes $p$ only; the current list is $2 \leqslant p \leqslant 43$ but $p \neq 23$, 31, or 41 .

Another question left open for $N>5$ is which pairs, triples, etc., of factors $\varkappa_{\xi}$ can appear simultaneously in the Alexander polynomial of a particular curve. This problem reduces to computing the genera of the intersections of the corresponding universal subgroups, including all their conjugates, or, equivalently, the genera of the connected components of the fibered products of their skeletons. We postpone this computation until the conjecture above has been settled.

\footnotetext{
${ }^{1}$ Added in proof: this conjecture has been proved in [12].
} 
It is worth mentioning that none of the groups $\bar{G}$ listed in Tables 1 and 3 is a congruence subgroup of $\Gamma$ (which is easily shown using the 'signatures' listed and the tables found in [5]). This fact refutes my original expectation that the fundamental group of a non-isotrivial genuine trigonal curve might be controlled by congruence subgroups.

\subsection{Idea of the proof}

Modifying the classical Zariski-van Kampen theorem, see Theorem 3.4, one reduces the study of the fundamental group of a (generalized) trigonal curve $C$ to a question about its monodromy group $\mathfrak{I m}_{C}$, which is a subgroup of the braid group $\mathbb{B}_{3}$ (respectively, of its extension via the inner automorphisms of the free group $\mathfrak{F}$ ). Crucial is the fact that the projection of $\mathfrak{I m}_{C}$ to the modular group $\Gamma$ is a subgroup of genus zero, see [6] and Theorem 3.2, which imposes a very strong restriction on $\mathfrak{I m}_{C}$. The Alexander polynomial is controlled by the reduced Burau representation, see [4], [18] and Subsection 2.2, which is a $\mathbb{B}_{3}$-action on a certain universal Alexander $\Lambda$-module $\mathrm{A} \cong \Lambda \oplus \Lambda$. Then, there remains to describe the 'Burau congruence subgroups' $\left\{\beta \in \mathbb{B}_{3} \mid \beta=\mathrm{id} \bmod \mathcal{V}\right\}$, where $\mathcal{V} \subset \mathrm{A}$ is a fixed submodule, and select those that are of genus zero.

Unfortunately, no convenient description of the image of $\mathbb{B}_{3}$ in $\mathrm{Mat}_{2 \times 2}(\Lambda)$ seems to be known, and we take a more geometric approach. A subgroup $G \subset \mathbb{B}_{3}$ is represented by its skeleton Sk, see Subsection 2.4, which is a certain planar (in the genus zero case) bipartite ribbon graph. Then, in Section 4, we derive some local restrictions on the geometry of Sk necessary for the nonvanishing of the Alexander module. In Section 5, these local restrictions and the planarity condition (Euler's formula $\chi(S)=2$, where $S$ is the minimal supporting surface of Sk) are used to narrow $N$ down to the range $N \leqslant 26$ (or $N \leqslant 21$ if $p=0$ ). In this finite range, we use a computer aided analysis to improve the a priori bound on the number of 'small' regions of $\mathrm{Sk}$ and reduce it further to $N \leqslant 10$, with the exception of finitely many triples $\left(p, N, \varkappa_{\xi}\right), p>0$, see Corollary 5.10. For each exceptional triple, we compute the genus of the corresponding universal subgroup $G$ by a straightforward coset enumeration in the finite group $G L\left(2, \mathbb{K}_{p}\right)$, thus proving Theorem 1.3.

In Section 6 , the case $N \leqslant 5$ is reduced to congruence subgroups of $\Gamma$, allowing an easy classification of the Alexander modules. Then, for $p=0$, we eliminate the range $6 \leqslant N \leqslant 10$ and prove Theorem 1.2. (For $N=7$ and 9 , we have to use Maple to show that the corresponding universal subgroups are of infinite index.)

Sections 2 and 3 are preliminary: we introduce the groups used and the necessary technical tools and explain the relation between trigonal curves and subgroups of $\mathbb{B}_{3}$. Section 7 deals with the parabolic case $N=6$ : we discover an infinite series of non-congruence subgroups of genus zero with nontrivial extended Alexander module.

Remark 1.6. As an interesting by-product of this research, not quite related to the original problem, we discover that 'Burau congruence subgroups' described above behave quite differently from the conventional congruence subgroups of $\Gamma$ : there are finitely many subgroups for $N \leqslant 5$, infinitely many finite index subgroups, all of genus zero or one, for $N=6$, and the subgroups seem to be of infinite index 
for $N \geqslant 7$ (although formally the latter claim has only been proved for $N=7$ and 9). Apparently, this is due to the fact that the Burau representation on A/ $\Phi_{N}(-t)$ is highly nontransitive for $N \geqslant 7$.

Acknowledgements. I am grateful to A. Libgober for his helpful remarks and stimulating discussions of the subject. The final version of the manuscript was prepared during my sabbatical stay at l'Instutut des Hautes Études Scientifiques and Max-Planck-Institut für Mathematik; I would like to extend my gratitude to these institutions for their support and hospitality.

\section{The braid group}

In this section, we introduce the braid group $\mathbb{B}_{3}$ and related objects, the principal purpose being fixing the notation and terminology.

\subsection{The group $\mathbb{B}_{3}$}

Let $\mathfrak{F}=\left\langle\alpha_{1}, \alpha_{2}, \alpha_{3}\right\rangle$ be the free group on three generators. The braid group $\mathbb{B}_{3}$ can be defined as the group of automorphisms $\beta: \mathfrak{F} \rightarrow \mathfrak{F}$ with the following properties:

- each generator $\alpha_{i}$ is taken to a conjugate of a generator;

- the element $\rho:=\alpha_{1} \alpha_{2} \alpha_{3}$ remains fixed.

Recall, see [1], that $\mathbb{B}_{3}=\left\langle\sigma_{1}, \sigma_{2} \mid \sigma_{1} \sigma_{2} \sigma_{1}=\sigma_{2} \sigma_{1} \sigma_{2}\right\rangle$, the Artin generators $\sigma_{1}, \sigma_{2}$ acting on $\mathfrak{F}$ via

$$
\sigma_{1}: \alpha_{1} \mapsto \alpha_{1} \alpha_{2} \alpha_{1}^{-1}, \quad \alpha_{2} \mapsto \alpha_{1} ; \quad \sigma_{2}: \alpha_{2} \mapsto \alpha_{2} \alpha_{3} \alpha_{2}^{-1}, \quad \alpha_{3} \mapsto \alpha_{2} .
$$

Note that the set of Artin generators depends on the basis $\left\{\alpha_{1}, \alpha_{2}, \alpha_{3}\right\}$.

In the sequel, we reserve the notation $\mathfrak{F}$ for the free group supplied with a $\mathbb{B}_{3}$-action, or, equivalently, with a distinguished set of bases constituting a whole $\mathbb{B}_{3}$-orbit. Any basis in the distinguished orbit is called geometric; any such basis gives rise to a pair of Artin generators of $\mathbb{B}_{3}$. We will also consider the degree homomorphisms

$$
\operatorname{deg}: \mathfrak{F} \rightarrow \mathbb{Z}, \quad \alpha_{1}, \alpha_{2}, \alpha_{3} \mapsto 1, \quad \operatorname{dg}: \mathbb{B}_{3} \rightarrow \mathbb{Z}, \quad \sigma_{1}, \sigma_{2} \mapsto 1 .
$$

It is straightforward that they do not depend on the choice of a geometric basis $\left\{\alpha_{1}, \alpha_{2}, \alpha_{3}\right\}$ and that for any $\alpha \in \mathfrak{F}, \beta \in \mathbb{B}_{3}$ one has $\operatorname{deg} \beta(\alpha)=\operatorname{deg} \alpha$.

With generalized trigonal curves in mind, see Subsection 3.3, we introduce also the extended group $\mathbb{B}_{3} \cdot \operatorname{Inn} \mathfrak{F} \subset$ Aut $\mathfrak{F}$, where $\operatorname{Inn} \mathfrak{F} \cong \mathfrak{F}$ is the subgroup of inner automorphisms of $\mathfrak{F}$. The intersection $\mathbb{B}_{3} \cap \operatorname{Inn} \mathfrak{F}$ is the cyclic group generated by $\left(\sigma_{2} \sigma_{1}\right)^{3}=\rho$; hence the degree map extends to the product via $\operatorname{dg}(\beta \cdot \alpha)=$ $\operatorname{dg} \beta+2 \operatorname{deg} \alpha$, where $\beta \in \mathbb{B}_{3}$ and $\alpha \in \operatorname{Inn} \mathfrak{F} \cong \mathfrak{F}$.

The natural action of $\mathbb{B}_{3} \cdot \operatorname{Inn} \mathfrak{F}$ on the set of conjugacy classes of geometric generators defines an epimorphism $\mathbb{B}_{3} \cdot \operatorname{Inn} \mathfrak{F} \rightarrow \mathbb{S}_{3}$. A subgroup $G \subset \mathbb{B}_{3} \cdot \operatorname{Inn} \mathfrak{F}$ is said to be $\mathbb{S}$-transitive if this action, restricted to $G$, is transitive. Clearly, $G$ is $\mathbb{S}$-transitive if and only if its image under the above epimorphism contains a cycle of length three. 
Given two subgroups $G$ and $H$ of $\mathbb{B}_{3}$ or $\mathbb{B}_{3} \cdot \operatorname{Inn} \mathfrak{F}$ (or any of the quotients $\mathrm{Bu}_{3}, \tilde{\Gamma}$, or $\Gamma$ considered below), we write $G \sim H$ if $G$ is conjugate to $H$ and $G \prec H$ if $G$ is subconjugate to $H$, i.e., if $G$ is conjugate to a subgroup of $H$.

\subsection{The Burau representation}

Denote by A the abelianization of the kernel Ker deg, and let $[h] \in$ A be the class of an element $h \in$ Ker deg. An element $\alpha \in \mathfrak{F}$ of degree one defines a homomorphism $t: \mathrm{A} \rightarrow \mathrm{A},[h] \mapsto\left[\alpha h \alpha^{-1}\right]$, which does not depend on $\alpha$. Thus, A turns into a module over the ring $\Lambda:=\mathbb{Z}\left[t, t^{-1}\right]$ of Laurent polynomials. An easy computation shows that $\mathrm{A}=\Lambda \mathbf{e}_{1} \oplus \Lambda \mathbf{e}_{2}$, where $\mathbf{e}_{1}=\left[\alpha_{2} \alpha_{1}^{-1}\right], \mathbf{e}_{2}=\left[\alpha_{3} \alpha_{2}^{-1}\right]$ in some geometric basis $\left\{\alpha_{1}, \alpha_{2}, \alpha_{3}\right\}$.

Since the $\mathbb{B}_{3}$-action on $\mathfrak{F}$ preserves the degree, it restricts to a certain action on A, which is called the (reduced) Burau representation, see [4]. This representation is faithful; for this reason we identify an element $\beta \in \mathbb{B}_{3}$ and the matrix in $\operatorname{Mat}_{2 \times 2}(\Lambda)$ representing it. The Artin generators $\sigma_{1}$ and $\sigma_{2}$ corresponding to the chosen geometric basis $\left\{\alpha_{1}, \alpha_{2}, \alpha_{3}\right\}$ (the one used to define $\mathbf{e}_{1}$ and $\mathbf{e}_{2}$ ) act via

$$
\sigma_{1}=\left[\begin{array}{cc}
-t & 1 \\
0 & 1
\end{array}\right], \quad \sigma_{2}=\left[\begin{array}{cc}
1 & 0 \\
t & -t
\end{array}\right]
$$

and the powers of these matrices are given by

$$
\sigma_{1}^{m}=\left[\begin{array}{cc}
(-t)^{m} & \tilde{\varphi}_{m}(-t) \\
0 & 1
\end{array}\right], \quad \sigma_{2}^{m}=\left[\begin{array}{cc}
1 & 0 \\
t \tilde{\varphi}_{m}(-t) & (-t)^{m}
\end{array}\right],
$$

where $\tilde{\varphi}_{m}(t):=\left(t^{m}-1\right) /(t-1)$. For future reference, observe that, for any $r \in \mathbb{Z}$, one has

$$
(t+1) t^{r} \tilde{\varphi}_{m}(-t)+t^{r}(-t)^{m}=t^{r}
$$

The following two matrices are also used in the sequel:

$$
\sigma_{2} \sigma_{1}=\left[\begin{array}{cc}
-t & 1 \\
-t^{2} & 0
\end{array}\right], \quad \sigma_{2} \sigma_{1} \sigma_{2}=\left[\begin{array}{cc}
0 & -t \\
-t^{2} & 0
\end{array}\right]
$$

The Burau representation extends to the product $\mathbb{B}_{3} \cdot \operatorname{Inn} \mathfrak{F}$. Clearly, the map Inn $\mathfrak{F}=\mathfrak{F} \rightarrow \operatorname{Mat}_{2 \times 2}(\Lambda)$ is given by $\alpha \mapsto t^{\operatorname{deg} \alpha}$ id. The image of $\mathbb{B}_{3} \cdot \operatorname{Inn} \mathfrak{F}$ in the group $G L(2, \Lambda)$ is denoted by $\mathrm{Bu}_{3}$; it is the central product $\mathbb{B}_{3} \odot \mathbb{Z}$, obtained by identifying the center $Z\left(\mathbb{B}_{3}\right)$ and the subgroup $3 \mathbb{Z} \subset \mathbb{Z}$ (both subgroups being generated by $\left.t^{3} \mathrm{id}\right)$. The center $Z\left(\mathrm{Bu}_{3}\right)$ is the cyclic subgroup formed by all scalar matrices $t^{r}$ id. The degree map dg descends to $\mathrm{Bu}_{3}$ and coincides, essentially, with the determinant: one has $\operatorname{det} \beta=(-t)^{\operatorname{dg} \beta}$ for any $\beta \in \mathrm{Bu}_{3}$.

Given two submodules $\mathcal{U}, \mathcal{V} \subset \mathrm{A}$, we say that $\mathcal{U}$ is conjugate to $\mathcal{V}, \mathcal{U} \sim \mathcal{V}$, if $\mathcal{V}=\beta(\mathcal{U})$ for some $\beta \in \mathbb{B}_{3}$, and $\mathcal{U}$ is subconjugate to $\mathcal{V}, \mathcal{U} \prec \mathcal{V}$, if $\mathcal{U}$ is conjugate to a submodule of $\mathcal{V}$. Clearly, in this definition $\mathbb{B}_{3}$ can be replaced with $\mathrm{Bu}_{3}$.

For an ideal $I \subset \Lambda$, we will use the notation $\mathcal{U} \sim \mathcal{V} \bmod I$ and $\mathcal{U} \prec \mathcal{V} \bmod I$ meaning the images of the modules in A/I. If $I=\Lambda f, f \in \Lambda$, is a principal ideal, we abbreviate $\bmod \Lambda f$ to $\bmod f$. 


\subsection{The modular representation}

Specializing all matrices at $t=-1$, one obtains homomorphisms $\mathbb{B}_{3}, \mathrm{Bu}_{3} \rightarrow \tilde{\Gamma}:=$ $S L(2, \mathbb{Z})$, which give rise to the modular representation

$$
\operatorname{pr}_{\Gamma}: \mathbb{B}_{3}, \mathrm{Bu}_{3} \rightarrow \Gamma:=\operatorname{PSL}(2, \mathbb{Z})=\tilde{\Gamma} / \pm \mathrm{id} .
$$

Usually, we abbreviate $\operatorname{pr}_{\Gamma} G=\bar{G}$ and $\operatorname{pr}_{\Gamma} \beta=\bar{\beta}$ for a subgroup $G \subset \mathrm{Bu}_{3}$ or an element $\beta \in \mathrm{Bu}_{3}$.

Recall that the modular group $\Gamma$ is generated by two elements $\mathbb{X}$ and $\mathbb{Y}$ subject to the relations $\mathbb{X}^{3}=\mathbb{Y}^{2}=1$. One can take $\mathbb{X}=\left(\bar{\sigma}_{2} \bar{\sigma}_{1}\right)^{-1}$ and $\mathbb{Y}=\bar{\sigma}_{2} \bar{\sigma}_{1}^{2}$; then $\bar{\sigma}_{1}=\mathbb{X} \mathbb{Y}$ and $\bar{\sigma}_{2}=\mathbb{X}^{2} \mathbb{Y} \mathbb{X}^{-1}$.

A subgroup of $\Gamma$ is called a congruence subgroup of level $l \mid n$ if it contains the principal congruence subgroup $\Gamma(n)=\{g \in \Gamma \mid g=\mathrm{id} \bmod n\}$. We make use of the list of congruence subgroups found in [5]; when referring to such subgroups, we use the notation of [5] and, whenever available, the alternative conventional notation.

The degree homomorphisms dg: $\mathbb{B}_{3} \rightarrow \mathbb{Z}$ and $\mathrm{dg}: \mathrm{Bu}_{3} \rightarrow \mathbb{Z}$ descend to well defined homomorphisms dg: $\Gamma \rightarrow \mathbb{Z}_{6}$ and $\operatorname{dg} \bmod 2: \Gamma \rightarrow \mathbb{Z}_{2}$, respectively. Thus, one has $\mathbb{B}_{3}=\Gamma \times_{\mathbb{Z}_{6}} \mathbb{Z}$ and $\mathrm{Bu}_{3}=\Gamma \times_{\mathbb{Z}_{2}} \mathbb{Z}$.

Definition 2.3. The depth $\operatorname{dp} G$ of a subgroup $G \subset \mathrm{Bu}_{3}$ is the degree of the positive generator of the intersection $G \cap \mathrm{Ker} \mathrm{pr}_{\Gamma}$, or zero if this intersection is trivial. One has $\operatorname{dp} G=0 \bmod 2$ and $\operatorname{dp} G=0 \bmod 6$ if $G \subset \mathbb{B}_{3}$.

Consider a subgroup $G \subset \mathrm{Bu}_{3}$, let $2 d=\operatorname{dp} G$, and let $G_{d}$ be the image of $G$ under the projection $\operatorname{pr}_{d}:=\operatorname{pr}_{\Gamma} \times(\operatorname{dg} \bmod 2 d): \mathrm{Bu}_{3} \rightarrow \Gamma \times \mathbb{Z}_{2 d}$. (We let $\mathbb{Z}_{0}=\mathbb{Z}$.) Then $G=\operatorname{pr}_{d}^{-1} G_{d}$ and $G_{d}$ projects isomorphically onto $\bar{G}$; in other words, $G_{d}$ is the graph of a certain homomorphism $\varphi: \bar{G} \rightarrow \mathbb{Z}_{2 d}$. This construction is summarized by the following definition and proposition, which is immediate.

Definition 2.4. The homomorphism $\varphi: \bar{G} \rightarrow \mathbb{Z}_{2 d}$ as above is called the slope of a subgroup $G \subset \mathrm{Bu}_{3}$.

Proposition 2.5. There is a one-to-one correspondence between the set of subgroups $G \subset \mathrm{Bu}_{3}$ and the set of pairs $(\bar{G}, \varphi)$, where $\bar{G} \subset \Gamma$ is a subgroup and $\varphi$ is a homomorphism $\bar{G} \rightarrow \mathbb{Z}_{2 d}$ with the property $\varphi=\operatorname{dg} \bmod 2$. One has $G \subset \mathbb{B}_{3}$ if and only if $d=0 \bmod 3$ and $\varphi=\operatorname{dg} \bmod 6$.

Each subgroup $\bar{G} \subset \Gamma$ admits three canonical slopes, namely, the restrictions to $\bar{G}$ of the homomorphisms $\pm \mathrm{dg}: \Gamma \rightarrow \mathbb{Z}_{6}$ and $\operatorname{dg} \bmod 2: \Gamma \rightarrow \mathbb{Z}_{2}$. We denote the corresponding subgroups of $\mathrm{Bu}_{3}$ by $(\bar{G})^{ \pm}$and $(\bar{G})^{\text {bu }}$, respectively. The subgroups $(\bar{G})^{\text {bu }}=\operatorname{pr}_{\Gamma}^{-1} \bar{G}$ and $(\bar{G})^{+}=(\bar{G})^{\text {bu }} \cap \mathbb{B}_{3}$ are merely the full preimages of $\bar{G}$ under $\operatorname{pr}_{\Gamma}: \mathrm{Bu}_{3} \rightarrow \Gamma$ and $\operatorname{pr}_{\Gamma}: \mathbb{B}_{3} \rightarrow \Gamma$, respectively.

\subsection{Skeletons}

In this subsection, we outline the relation between subgroups of $\Gamma$ and certain bipartite ribbon graphs, called skeletons. This and other very similar constructions have been studied, e.g., in [2], [3], and [14]. In the exposition below we follow the recent paper [11], where all proofs and further details can be found. 
Recall that a bipartite graph is a graph whose vertices are divided into two kinds, -- and o-, so that the two ends of each edge are of the opposite kinds. A ribbon graph is a graph equipped with a distinguished cyclic order (i.q., transitive $\mathbb{Z}$ action) on the star of each vertex. Any graph embedded in an oriented surface $S$ is a ribbon graph, with the cyclic order induced from the orientation of $S$. Conversely, any finite ribbon graph defines a unique, up to homeomorphism, closed oriented surface $S$ into which it is embedded: the star of each vertex is embedded in a small oriented disk (it is this step where the cyclic order is used), these disks are connected by oriented ribbons along edges producing a tubular neighborhood of the graph, and finally each boundary component of the resulting compact surface is patched with a disk. (Intuitively, the boundary components patched at the last step are the regions defined combinatorially in Subsection 2.4.3 below.) The surface $S$ thus constructed is called the minimal supporting surface of the ribbon graph.

In the rest of this section, we redefine a certain class of bipartite ribbon graphs in purely combinatorial terms, relating them to the modular group. In spite of this combinatorial approach, we will freely use the topological language applicable to the geometric realizations of the graphs.

2.4.1. The skeleton of a subgroup. Given a subgroup $G \subset \Gamma$, its skeleton $\mathrm{Sk}=\mathrm{Sk}_{G}$ is the bipartite ribbon graph, possibly infinite, defined as follows: the set of edges of Sk is the $\Gamma$-set $\Gamma / G$, its $\bullet$ - and o-vertices are the orbits of $\mathbb{X}$ and $\mathbb{Y}$, respectively, and the cyclic order (ribbon graph structure) at a trivalent $\bullet$-vertex is given by $\mathbb{X}^{-1}$. (All other vertices are at most bivalent and cyclic order is irrelevant.) The skeleton $\mathrm{Sk}_{G}$ is equipped with a distinguished edge, namely the coset $G / G$.

By definition, Sk is a connected bipartite graph with the following properties:

- the valency of each $\bullet$-vertex equals 1 or 3 (a divisor of ord $\mathbb{X}=3$ ), and

- the valency of each o-vertex equals 1 or 2 (a divisor of ord $\mathbb{Y}=2$ ).

Conversely, the set of edges of any connected bipartite ribbon graph Sk satisfying the valency restriction above admits a natural structure of a transitive $\Gamma$-set (the actions of $\mathbb{X}^{-1}$ and $\mathbb{Y}$ following the cyclic order at the $\bullet$ - and o-vertices, respectively), and the original subgroup $G$ can be recovered, up to conjugation, as the stabilizer $\operatorname{Stab}(e)$ of any edge $e$ of Sk.

Convention 2.6. In the figures, we omit bivalent o-vertices, assuming that such a vertex is to be inserted at the center of each edge connecting two •-vertices. With an abuse of the language, we will speak about adjacent •-vertices, meaning that they are connected by a pair of edges with a common bivalent o-vertex.

As usual, skeletons of genus zero (see Subsection 2.4.3 below) are drawn in the disk, assuming the blackboard thickening for the ribbon graph structure. The boundary of the disk (the dotted grey circle in the figures) represents a single point in the sphere $S^{2}$.

2.4.2. Paths. Topologically, it is convenient to regard $\mathrm{Sk}$ as an orbifold, assigning to each monovalent •- or o-vertex ramification index 3 or 2 , respectively. Then there is a canonical isomorphism

$$
G=\operatorname{Stab}(e)=\pi_{1}^{\mathrm{orb}}(\mathrm{Sk}, e),
$$


where the basepoint for the fundamental group is chosen inside an edge $e$. In fact, homotopy classes of paths in Sk (taking into account the orbifold structure) can be identified with pairs $\left(e_{0}, g\right)$, where the starting point $e_{0}$ is an edge and $g \in \Gamma$; the ending point of such a path is then $e_{1}:=g^{-1} e_{0}$. Intuitively, one starts at $e_{0}$ and constructs a path edge by edge, choosing at each intermediate step one of the four possible directions: turning about the $\bullet$ - or o-end of the last edge in the positive or negative direction (with respect to the distinguished cyclic order); these directions are encoded by the letters $\mathbb{Y}^{-1}=\mathbb{Y}$ or $\mathbb{X}^{\mp 1}$ in the word representing $g$.

A path $(e, g), g \in \Gamma$, is a loop if and only if $e=g^{-1} e$, i.e., $g \in \operatorname{Stab}(e)$; hence the isomorphism above.

2.4.3. Regions. A region of a skeleton Sk is an orbit of $\mathbb{X} \mathbb{Y}$. The cardinality of a region $R$ is called its width wd $R$. (In the arithmetical theory, instead of regions one speaks about cusps and cusp widths; this, and the fact that the term 'degree' is overused, explains the terminology.) A region $R$ of width $n$ is also referred to as an $n$-gon or $n$-gonal region, 'corners' being the $\bullet$-vertices in the boundary of $R$. If Sk is finite, then, patching each region with an oriented disk, one obtains a minimal compact oriented surface $S$ supporting Sk. Its genus is called the genus of Sk and of the subgroup $G \subset \Gamma$ corresponding to Sk. (This definition is equivalent to the conventional one, see [11].) Using the projection $\mathrm{pr}_{\Gamma}$, we extend the notions of skeleton, genus, etc., to subgroups of $\mathrm{Bu}_{3}$.

A marking at a trivalent $\bullet$-vertex $v$ is a choice of an edge $e$ adjacent to $v$. The region (orbit) containing an edge $e$ is denoted by $((e))$. Thus, the three regions adjacent to a marked vertex $(v, e)$ are $((e)),((\mathbb{X} e))$, and $\left(\left(\mathbb{X}^{2} e\right)\right)$. By default, given a region $R$, a marking $e$ at each vertex $v$ in $\partial R$ is chosen so that $R=((e))$. Note that a vertex may appear in $\partial R$ more then once; in this case each occurrence gets its own marking.

2.4.4. Coverings. An inclusion $G^{\prime} \subset G$ of two subgroups gives rise to a $\Gamma$-map $\mathrm{Sk}^{\prime} \rightarrow$ Sk of their skeletons, which is a covering with respect to the orbifold structure defined in Subsection 2.4.2. It extends to an essentially unique (ramified) covering $S^{\prime} \rightarrow S$ of the minimal surfaces, see Subsection 2.4.3. The covering $\mathrm{Sk}^{\prime} \rightarrow \mathrm{Sk}$ is called (un-)ramified if $S^{\prime} \rightarrow S$ is (un-)ramified. In other words, the covering is unramified if and only if the pullback of each monovalent vertex of Sk consists of monovalent vertices only and the pullback of each region $R$ of Sk consists of regions of the same width wd $R$.

2.4.5. Canonical bases. In the definition of the skeleton $\mathrm{Sk}$ of a subgroup $G$, we use a distinguished pair $\mathbb{X}, \mathbb{Y}$ of generators of $\Gamma$, hence a distinguished pair $\sigma_{1}, \sigma_{2}$ of Artin generators of $\mathbb{B}_{3}$, hence a distinguished geometric basis $\left\{\alpha_{1}, \alpha_{2}, \alpha_{3}\right\}$ of $\mathfrak{F}$; the latter is defined up to the action of the center $Z\left(\mathbb{B}_{3}\right)$, i.e., up to conjugation by $\rho$.

One has $G=\pi_{1}^{\text {orb }}(\mathrm{Sk}, e)$, where $e=G / G$ is the distinguished edge of Sk, see Subsection 2.4.2. If $e^{\prime}$ is another edge, we fix a path $\gamma=(e, g)$ from $e$ to $e^{\prime}$ and identify $\pi_{1}^{\text {orb }}\left(\mathrm{Sk}, e^{\prime}\right)$ with $G$ via the translation isomorphism $\delta \mapsto \gamma \delta \gamma^{-1}$, i.e., via 
the conjugation by $g$. Alternatively, one can lift $g$ to an element $\tilde{g} \in \mathbb{B}_{3}$ and consider the new geometric basis $\left\{\alpha_{1}^{\prime}, \alpha_{2}^{\prime}, \alpha_{3}^{\prime}\right\}, \alpha_{i}^{\prime}=\tilde{g}\left(\alpha_{i}\right)$, for $\mathfrak{F}$. In this sense, assuming $\gamma$ fixed, we will speak of a canonical basis over $e^{\prime}$.

\subsection{Type specification}

If $G \subset \mathrm{Bu}_{3}$ is a subgroup of genus zero, its slope can be described in terms of its skeleton Sk. In view of Subsection 2.4.2, the projection $\bar{G} \subset \Gamma$ has a presentation of the form

$$
\left\langle\beta_{R}, \gamma_{v} \mid\left(\gamma_{v}^{\bullet}\right)^{3}=\left(\gamma_{v}^{\circ}\right)^{2}=1, \prod \beta_{R} \prod \gamma_{v}=1\right\rangle
$$

where the indices $R$ and $v$ run, respectively, over all regions and monovalent vertices of Sk and the superscript indicates the type of the vertex. (The product in the last relation is ordered in a certain manner depending on the choice of the basis. In fact, $\left\{\beta_{R}, \gamma_{v}\right\}$ is merely a geometric basis for the fundamental group of a punctured sphere, $c f$. Definition 3.1 below.) Furthermore, each generator $\beta_{R}$ is conjugate to $\bar{\sigma}_{1}^{\text {wd } R}$, and each generator $\gamma_{v}$ is conjugate to $\mathbb{X}^{-1}=\bar{\sigma}_{2} \bar{\sigma}_{1}$ or $\mathbb{Y}=\bar{\sigma}_{2} \bar{\sigma}_{1}^{2}$, depending on whether $v$ is a $\bullet$ - or o-vertex.

Definition 2.8. The type specification of a subgroup $G \subset \mathrm{Bu}_{3}$ of genus zero is the

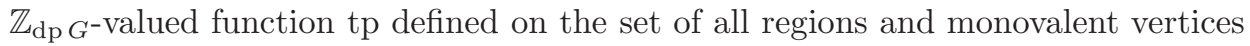
of the skeleton $\mathrm{Sk}_{G}$; each region or monovalent vertex is sent to the degree of (any) lift to $G$ of the corresponding generator in (2.7) or, equivalently, to the value of the slope of $G$ on the corresponding generator.

Proposition 2.9. Let $d=6$ if $G \subset \mathbb{B}_{3}$ and $d=2$ otherwise. Then one has:

1. $\operatorname{dp} G=0 \bmod d ;$

2. $\operatorname{tp}(R)=\operatorname{wd} R \bmod d$ for any region $R$;

3. $\operatorname{tp}(\bullet)=2 \bmod d$ and $3 \operatorname{tp}(\bullet)=0$;

4. $\operatorname{tp}(\circ)=3 \bmod d$ and $2 \operatorname{tp}(\circ)=0$;

5. the sum of all values of tp equals zero.

Any pair (dp, tp) satisfying (1)-(5) above defines a unique slope; such a pair results in a subgroup $G \subset \mathbb{B}_{3}$ if and only if it satisfies (1)-(4) with $d=6$.

Proof. The $(\bmod d)$-congruences in $(1)-(4)$ follow from the properties of slopes, see Proposition 2.5, and the other relations in (3)-(5) are the abelian versions of the relations in (2.7). The type specification determines the slope of $G$ as it assigns a value to each generator in (2.7).

Given an integer $m$, a type specification is said to be trivial modulo $m$ if it satisfies the congruences in (1)-(4) of Proposition 2.9 with $d=m$. Thus, Proposition 2.9 states that any type specification is trivial modulo 2 and that a subgroup $G$ is in $\mathbb{B}_{3}$ if and only if its type specification is trivial modulo 6 .

Convention 2.10. In the drawings, we indicate the type specification (inside a region or next to a vertex) only when it is not trivial modulo 0 . 


\subsection{The Alexander module}

For a subgroup $G \subset \mathbb{B}_{3} \cdot \operatorname{Inn} \mathfrak{F}$, let

$$
\overline{\mathcal{V}}_{G}=\sum_{\beta \in G} \operatorname{Im}(\beta-\mathrm{id}) \subset \mathrm{A}, \quad \mathcal{V}_{G}=\sum_{\beta \in G, \alpha \in \mathfrak{F}} \Lambda\left[\beta(\alpha) \cdot \alpha^{-1}\right] \subset \mathrm{A} .
$$

Definition 2.11. The Alexander module of a subgroup $G \subset \mathbb{B}_{3} \cdot \operatorname{Inn} \mathfrak{F}$ is the $\Lambda$-module $\mathrm{A}_{G}:=\mathrm{A} / \mathcal{V}_{G}$. If the product $\mathrm{A}_{G} \otimes \mathbb{k}_{p}$ is a torsion $\left(\Lambda \otimes \mathbb{k}_{p}\right)$-module, its order $\Delta_{G, p} \in \Lambda \otimes \mathbb{k}_{p}$ is called the $(\bmod p)$-Alexander polynomial of $G$. We usually abbreviate $\Delta_{G, 0}=\Delta_{G}$.

Definition 2.12. The extended Alexander module of a subgroup $G \subset \mathbb{B}_{3} \cdot \operatorname{Inn} \mathfrak{F}$ is the $\Lambda$-module $\overline{\mathrm{A}}_{G}:=\mathrm{A} / \overline{\mathcal{V}}_{G}$; the extended Alexander polynomial $\bar{\Delta}_{G, p} \in \Lambda \otimes \mathbb{k}_{p}$ (whenever defined) is the order of the $\left(\Lambda \otimes \mathbb{k}_{p}\right)$-module $\overline{\mathrm{A}}_{G} \otimes \mathbb{k}_{p}$.

Clearly, the Alexander polynomial $\Delta_{G, p}$ and its extended counterpart $\bar{\Delta}_{G, p}$ can be computed using any field $\mathbb{K}$ of characteristic $p$, and the Alexander polynomial can be interpreted as the characteristic polynomial of the operator $t$ acting on the finite dimensional $\mathbb{K}$-vector space $\mathrm{A}_{G} \otimes \mathbb{K}$ (respectively, $\overline{\mathrm{A}}_{G} \otimes \mathbb{K}$ ).

Remark 2.13. Assume that $G=\mathfrak{I m}_{C}$ is the monodromy group of a trigonal curve, see Subsections 3.2 and 3.3 below. Then, the conventional Alexander module $\mathrm{A}_{G}$ is the Alexander module of $C$; it depends on the fundamental group of $C$ only, see Subsection 3.4. On the contrary, the submodule $\overline{\mathcal{V}}_{G} \subset \mathrm{A}$ depends only on the image of $G$ in $\mathrm{Bu}_{3}$; thus, it is easier to compute. Furthermore, $\overline{\mathcal{V}}_{G}, \overline{\mathrm{A}}_{G}$, and the extended Alexander polynomials can be defined for subgroups $G$ of $\mathrm{Bu}_{3}$ rather than those of the more complicated group $\mathbb{B}_{3} \cdot \operatorname{Inn} \mathfrak{F}$. There is a canonical epimorphism $\overline{\mathrm{A}}_{G} \rightarrow \mathrm{A}_{G}$, see Lemma 2.16, and the conventional Alexander polynomials divide their extended counterparts (whenever defined). For this reason, and since we are mainly interested in an upper bound on the Alexander polynomial, we will usually deal with the extended versions. Lemma 2.17 and Corollary 2.18 below show that, for subgroups of $\mathbb{B}_{3}$ (i.q., genuine trigonal curves), the two submodules $\overline{\mathcal{V}}_{G}, \mathcal{V}_{G} \subset \mathrm{A}$ usually coincide.

Definition 2.14. Given a submodule $\mathcal{V} \subset \mathrm{A}$, the set

$$
G_{\mathcal{V}}=\left\{\beta \in \mathrm{Bu}_{3} \mid \operatorname{Im}(\beta-\mathrm{id}) \subset \mathcal{V}\right\}
$$

is a subgroup of $\mathrm{Bu}_{3}, c f$. [6]; it is called the universal subgroup corresponding to $\mathcal{V}$.

Definitions 2.11, 2.12, and 2.14 have a geometric meaning for subgroups of genus zero, see Subsection 3.4 below. In general, it is not quite clear how the Alexander modules and, especially, universal subgroups should be defined, see Remark 3.6.

Next two statements are straightforward.

Lemma 2.15. For subgroups $G, H \subset \mathrm{Bu}_{3}$ and submodules $\mathcal{U}, \mathcal{V} \subset \mathrm{A}$, one has

1. if $G \prec H$, then $\overline{\mathcal{V}}_{G} \prec \overline{\mathcal{V}}_{H}$;

2. if $\mathcal{U} \prec \mathcal{V}$, then $G_{\mathcal{U}} \prec G_{\mathcal{V}}$;

3. $\overline{\mathcal{V}}_{G} \prec \mathcal{U}$ if and only if $G \prec G_{\mathcal{U}}$. 
Lemma 2.16. One has:

1. $\overline{\mathcal{V}}_{G} \subset \mathcal{V}_{G}$,

2. $\left[\beta(\alpha h) \cdot(\alpha h)^{-1}\right]=\left[\beta(\alpha) \cdot \alpha^{-1}\right]+t^{\operatorname{deg} \alpha}(\beta-\mathrm{id})[h]$ for any $h \in$ Ker deg,

3. $\left[\beta\left(\alpha^{n}\right) \cdot \alpha^{-n}\right]=\tilde{\varphi}_{n}\left(t^{\operatorname{deg} \alpha}\right)\left[\beta(\alpha) \cdot \alpha^{-1}\right]$ for any $n \in \mathbb{Z}$,

where $\beta \in \mathbb{B}_{3}$ and $\alpha \in \mathfrak{F}$. As a consequence,

4. $\mathcal{V}_{G}=\overline{\mathcal{V}}_{G}+\sum_{\beta \in G} \Lambda\left[\beta\left(\alpha_{i}\right) \cdot \alpha_{i}^{-1}\right]$ for any geometric generator $\alpha_{i} \in \mathfrak{F}$.

Lemma 2.17. For a subgroup $G \in \mathbb{B}_{3}$, one has $\left(t^{2}+t+1\right) \mathcal{V}_{G} \subset \overline{\mathcal{V}}_{G}$.

Proof. Since $\operatorname{deg} \alpha_{1}^{3}=3=\operatorname{deg} \rho$, for any braid $\beta \in \mathbb{B}_{3}$ one has

$$
\left(t^{2}+t+1\right)\left[\beta\left(\alpha_{1}\right) \cdot \alpha_{1}^{-1}\right]=\left[\beta\left(\alpha_{1}^{3}\right) \cdot \alpha_{1}^{-3}\right]=\left[\beta(\rho) \cdot \rho^{-1}\right] \bmod \overline{\mathcal{V}}_{G},
$$

see Lemma $2.16(3)$ and (2). Since $\rho$ is $\mathbb{B}_{3}$-invariant, this expression is $0 \bmod \overline{\mathcal{V}}_{G}$, and the statement follows from Lemma $2.16(4)$.

Corollary 2.18. For any subgroup $G \subset \mathbb{B}_{3}$, field $\mathbb{K}$, and polynomial $f \in \Lambda \otimes \mathbb{K}$ prime to $t^{2}+t+1$, the images of $\overline{\mathcal{V}}_{G}$ and $\mathcal{V}_{G}$ in $(\mathrm{A} \otimes \mathbb{K}) / f$ coincide.

\subsection{Specializations}

Recall that we write $\mathbb{k}_{0}=\mathbb{Q}$ and $\mathbb{k}_{p}=\mathbb{F}_{p}$ for $p$ prime. If $p$ is understood, we drop the index. The notation $\varkappa_{\xi} \in \mathbb{k}[t]$ stands for the minimal polynomial of an element $\xi \neq 0$ of an algebraic extension $\mathbb{K} \supset \mathbb{k}$.

Definition 2.19. The multiplicative order of an element $\xi \in \mathbb{K}^{*}$ is denoted by ord $\xi$. (If $\xi$ is not a root of unity, we let ord $\xi=\infty$.) For $N \in \mathbb{Z}_{+}$not divisible by $p$ (where $p$ is a prime or zero), define $\mathrm{e}_{p}(N)$ as follows: $\mathrm{e}_{2}(N)=N$ and

$$
\mathrm{e}_{p}(N)= \begin{cases}2 N, & \text { if } N=1 \bmod 2, \\ \frac{1}{2} N, & \text { if } N=2 \bmod 4, \\ N, & \text { if } N=0 \bmod 4\end{cases}
$$

for $p \neq 2$. Then $\mathrm{e}_{p}(\operatorname{ord} \xi)=\operatorname{ord}(-\xi)$ and $\mathrm{e}_{p}$ is an involution: $\mathrm{e}_{p}\left(\mathrm{e}_{p}(N)\right)=N$.

Given $\xi$ as above, we define the specializations of $\Lambda$ and $\mathrm{A}$ at $\xi$ to be $\Lambda(\xi)=$ $(\Lambda \otimes \mathbb{k}) / \varkappa_{\xi}$ and $\mathrm{A}(\xi)=(\mathrm{A} \otimes \mathbb{k}) / \varkappa_{\xi}$, respectively. (The specializations of other relevant modules are defined below on a case-by-case basis.) Usually we assume that $\mathbb{K}=\Lambda(\xi)$; then $\mathrm{A}(\xi)$ is a $\mathbb{K}$-vector space of dimension 2 .

For a subgroup $G \subset \mathbb{B}_{3} \cdot \operatorname{Inn} \mathfrak{F}$, define the specializations $\overline{\mathcal{V}}_{G}(\xi) \subset \mathcal{V}_{G}(\xi) \subset \mathrm{A}(\xi)$ as the images of $\overline{\mathcal{V}}_{G} \otimes \mathbb{k}$ and $\mathcal{V}_{G} \otimes \mathbb{k}$ in $\mathrm{A}(\xi)$, respectively. (In general, the maps $\overline{\mathcal{V}}_{G} \otimes \mathbb{k} \rightarrow \mathrm{A} \otimes \mathbb{k}$ are not monomorphisms.) As above, these images can be regarded as $\mathbb{K}$-vector subspaces. If $G \subset \mathbb{B}_{3}$ and $\xi^{2}+\xi+1 \neq 0$, these two subspaces coincide, see Corollary 2.18. We write $\overline{\mathrm{A}}_{G}(\xi)=\mathrm{A}(\xi) / \overline{\mathcal{V}}_{G}(\xi)$ and $\mathrm{A}_{G}(\xi)=\mathrm{A}(\xi) / \mathcal{V}_{G}(\xi)$. The barred versions of all objects can also be defined for a subgroup $G \subset \mathrm{Bu}_{3}$.

We extend the notion of (sub-)conjugacy, see Subsection 2.2, and the notation and $\prec$ to submodules of $\mathrm{A} \otimes \mathbb{k}$ and $\mathrm{A}(\xi)$. The concept of universal subgroup, see Definition 2.14, can also be extended to submodules of $\mathrm{A} \otimes \mathbb{k}$ and $\mathrm{A}(\xi)$, and an analog of Lemma 2.15 holds literally. 


\section{Trigonal curves}

In this section, we introduce (generalized) trigonal curves and their monodromy groups. Proofs are mostly omitted; for all details see [6] and references therein.

\subsection{Trigonal curves in Hirzebruch surfaces}

A Hirzebruch surface $\Sigma_{d}$ is a geometrically ruled rational surface with an exceptional section $E$ of self-intersection $-d \leqslant 0$. The fibers of $\Sigma_{d}$ are the fibers of the ruling $\Sigma_{d} \rightarrow \mathbb{P}^{1}$. To avoid excessive notation, we identify fibers and their images in the base $\mathbb{P}^{1}$. The semigroup of classes of effective divisors on $\Sigma_{d}$ is freely generated by the classes $|E|$ and $|F|$, where $F$ is any fiber.

A generalized trigonal curve is a reduced curve $C \subset \Sigma_{d}$ intersecting each fiber at three points, counted with multiplicities; in other words, $C \in|3 E+3 d F|$. A (genuine) trigonal curve is a generalized trigonal curve disjoint from the exceptional section $E \subset \Sigma_{d}$. A singular fiber of a generalized trigonal curve $C \subset \Sigma_{d}$ is a fiber $F$ of $\Sigma_{d}$ intersecting $C \cup E$ geometrically at fewer than four points, i.e., such that either $C$ is tangent to $F$ or the union $C \cup E$ has a singular point in $F$.

We emphasize that, from our point of view, a trigonal curve is always a curve embedded in a certain way in a certain Hirzebruch surface; the latter is assumed even if it is not mentioned explicitly. In particular, all (iso-, auto-, etc.) morphisms of trigonal curves are supposed to extend to their respective surfaces.

The (functional) $j$-invariant $j_{C}: \mathbb{P}^{1} \rightarrow \mathbb{P}^{1}$ of a trigonal curve $C \subset \Sigma_{d}$ is the analytic continuation of the function sending a nonsingular fiber $F$ to the $j$-invariant (divided by $12^{3}$ ) of the elliptic curve covering $F$ and ramified at $F \cap(C \cup E)$. In appropriate affine coordinates $(x, y)$ in $\Sigma_{d}$ ( $\operatorname{such}$ that $\left.E=\{y=\infty\}\right)$ the curve $C$ can be given by its Weierstraß equation

$$
y^{3}+3 p(x) y+2 q(x)=0 .
$$

Then

$$
j_{C}(x)=\frac{p^{3}}{\Delta}, \quad \text { where } \quad \Delta(x)=p^{3}+q^{2} .
$$

The curve $C$ is called isotrivial if $j_{C}=$ const. A non-isotrivial trigonal curve $C$ is determined by its $j$-invariant up to Nagata equivalence, see below.

A positive (negative) Nagata transformation is the birational transformation $\Sigma_{d} \rightarrow \Sigma_{d \pm 1}$ consisting in blowing up a point $P$ on (respectively, not on) the exceptional section $E$ and blowing down the proper transform of the fiber through $P$. An $m$-fold Nagata transformation is a sequence of $m$ Nagata transformations of the same sign over the same point of the base. Two trigonal curves $C$ and $C^{\prime}$ are called $m$-Nagata equivalent if $C^{\prime}$ is the proper transform of $C$ under a sequence of $m$-fold Nagata transformations. The special case $m=1$ is referred to simply as Nagata equivalence.

Each generalized trigonal curve $C$ is Nagata equivalent to a genuine one, which is unique up to Nagata equivalence. It is called a trigonal model of $C$.

Given a nonconstant holomorphic map $\tilde{\jmath}: \mathbb{P}^{1} \rightarrow \mathbb{P}^{1}$, the ruled surface $\Sigma^{\prime}:=$ $\tilde{\jmath}^{*} \Sigma_{d}$ is also a Hirzebruch surface; it is isomorphic to $\Sigma_{d \cdot \operatorname{deg} \tilde{\jmath}}$. Given a trigonal 
curve $C \subset \Sigma_{d}$, its divisorial pullback $C^{\prime}:=\tilde{\jmath}^{*} C \subset \Sigma^{\prime}$ is also a trigonal curve; it is said to be induced from $C$ by $\tilde{\jmath}$.

\subsection{Braid monodromy}

Introduced in this subsection are a few prerequisites for the classical Zariski-van Kampen theorem: we define the notion of proper section and, using such a section, construct the braid monodromy of a curve. The construction applies literally to any curve disjoint from the exceptional section; in the case of a trigonal curve $C \subset \Sigma_{d}$, it turns out that the monodromy group captures quite a few essential geometric properties of $C$. See Theorem 3.2 for the precise statement.

Fix a Hirzebruch surface $\Sigma_{d}$. For a fiber $F$ of $\Sigma_{d}$, the complement $F^{\circ}:=F \backslash E$ is an affine space over $\mathbb{C}$. Hence, one can speak of the convex hull of a subset of $F^{\circ}$. For a subset $S \subset \Sigma_{d} \backslash E$, denote by $\operatorname{conv}_{F} S$ the convex hull of $S \cap F^{\circ}$ in $F^{\circ}$ and let conv $S=\bigcup_{F} \operatorname{conv}_{F} S$.

Fix a genuine trigonal curve $C \subset \Sigma_{d}$. The term 'section' stands for a continuous section of (a restriction of) the fibration $p: \Sigma_{d} \rightarrow \mathbb{P}^{1}$. Let $\Delta \subset \mathbb{P}^{1}$ be a closed topological disk. (In what follows, we take for $\Delta$ the complement of a small regular neighborhood of a nonsingular fiber $F_{0} \in \mathbb{P}^{1}$.) A section $s: \Delta \rightarrow \Sigma_{d}$ of $p$ is called proper if its image is disjoint from both $E$ and conv $C$. As a simple consequence of the obstruction theory, any disk $\Delta \subset \mathbb{P}^{1}$ admits a proper section $s: \Delta \rightarrow \Sigma_{d}$, unique up to homotopy in the class of proper sections.

Fix a disk $\Delta \subset \mathbb{P}^{1}$ and let $F_{1}, \ldots, F_{r} \in \Delta$ be all the singular and, possibly, some nonsingular fibers of $C$ that belong to $\Delta$. Assume that all these fibers are in the interior of $\Delta$. Let $\Delta^{\circ}=\Delta \backslash\left\{F_{1}, \ldots, F_{r}\right\}$ and fix a reference fiber $F \in \Delta^{\circ}$. Then, given a proper section $s$, one can define the group $\pi_{F}:=\pi_{1}\left(F^{\circ} \backslash C, s(F)\right)$ and the braid monodromy, which is the anti-homomorphism $\mathfrak{m}: \pi_{1}\left(\Delta^{\circ}, F\right) \rightarrow$ Aut $\pi_{F}$ sending a loop $\gamma$ to the automorphism obtained by dragging $F$ along $\gamma$ and keeping the reference point in $s$.

Definition 3.1. Let $D$ be an oriented punctured disk, and let $b \in \partial D$. A geometric basis in $D$ is a basis $\left\{\gamma_{1}, \ldots, \gamma_{r}\right\}$ for the free group $\pi_{1}(D, b)$ formed by the classes of positively oriented lassoes about the punctures, pairwise disjoint except at the common reference point $b$, and such that $\gamma_{1} \ldots \gamma_{r}=[\partial D]$.

Shrink the reference fiber $F$ to a closed disk containing $\operatorname{conv}_{F} C$ in its interior and $s(F)$ in its boundary. Pick a geometric basis for $\pi_{F}$ and identify it with a geometric basis $\left\{\alpha_{1}, \alpha_{2}, \alpha_{3}\right\}$ for $\mathfrak{F}$, establishing an isomorphism $\pi_{F} \cong \mathfrak{F}$. Under this isomorphism, the braid monodromy $\mathfrak{m}$ takes values in the braid group $\mathbb{B}_{3} \subset$ Aut $\mathfrak{F}$. The monodromy $\mathfrak{m}$ thus defined is independent of the choice of a proper section, and another choice of the geometric bases for $\pi_{F}$ and $\mathfrak{F}$ results in the global conjugation by a fixed braid $\beta \in \mathbb{B}_{3}$, i.e., in the map $\gamma \mapsto \beta^{-1} \mathfrak{m}(\gamma) \beta$. Thus, the monodromy group $\mathfrak{I m}_{C}:=\operatorname{Im} \mathfrak{m} \subset \mathbb{B}_{3}$ is determined by $C$ up to conjugation. One has $\operatorname{dp} \mathfrak{I m}_{C} \mid 6 d$; the group $\mathfrak{I m}_{C}$ is $\mathbb{S}$-transitive if and only if $C$ is irreducible.

The next statement is proved in [6]. 
Theorem 3.2. The monodromy group of a non-isotrivial trigonal curve is of genus zero. Conversely, given a subgroup $G \subset \mathbb{B}_{3}$ of genus zero and depth $6 d>0$, there is a unique, up to isomorphism and $d$-Nagata equivalence, trigonal curve $C_{G}$ with the following property: for a non-isotrivial trigonal curve $C$ one has $\mathfrak{I m}_{C} \prec G$ if and only if $C$ is d-Nagata equivalent to a curve induced from $C_{G}$. This curve $C_{G}$ is called the universal curve corresponding to $G$.

The universal curve $C_{G}$ can be reconstructed from the skeleton $\mathrm{Sk}_{G}$. In fact, $\mathrm{Sk}_{G}$ is the dessin d'enfants, in the sense of Grothendieck, of a unique (up to Möbius transformation of the source) regular map $j: \mathbb{P}^{1} \rightarrow \mathbb{P}^{1}=\mathbb{C} \cup \infty$ with three critical values 0,1 , and $\infty$ only. This map $j$ is the $j$-invariant of $C_{G}$ (thus defining $C_{G}$ up to Nagata equivalence), and the types of the singular fibers of $C_{G}$ are given by the type specification of $G$ (which explains the term).

\subsection{Generalized curves}

Now, let $C \subset \Sigma_{d}$ be a generalized trigonal curve. This time, the closure of conv $C$ does not need to be compact and $C$ may not admit a proper section. To overcome this difficulty, consider a proper model $C^{\prime} \subset \Sigma_{d^{\prime}}$ of $C$ and, for a punctured disk $\Delta^{\circ}$ as above, let $\mathfrak{m}^{\prime}: \pi_{1}\left(\Delta^{\circ}, F\right) \rightarrow \mathbb{B}_{3}$ be the braid monodromy of $C^{\prime}$. Further, fix a geometric basis $\left\{\gamma_{1}, \ldots, \gamma_{r}\right\}$ for $\pi_{1}\left(\Delta^{\circ}, F\right)$. Then, the difference between $C$ and $C^{\prime}$ can be described in terms of the so-called slopes $\varkappa_{i} \in \mathfrak{F}$ assigned to each geometric generator $\gamma_{i}$. Roughly, assume that $\gamma_{i}$ is represented by a loop of the form $l_{i} \cdot \mu_{i} \cdot l_{i}^{-1}$, where $\mu_{i}$ is a small circle about a fiber $F_{i}$ and $l_{i}$ is a simple path connecting the common base point and a point $a_{i} \in \mu_{i}$. Consider a small analytic disk $\Phi \subset \Sigma_{d}$ transversal to $F_{i}$ and disjoint from $C$ and $E$, and a similar disk $\Phi^{\prime} \subset \Sigma_{d^{\prime}}$ with respect to $C^{\prime}$. Let $\bar{\Phi} \subset \Sigma_{d^{\prime}}$ be the transform of $\Phi$, and assume that the boundaries $\partial \Phi^{\prime}$ and $\partial \bar{\Phi}$ have a common point over $a_{i}$. Then, the loop $[\partial \bar{\Phi}] \cdot\left[\partial \Phi^{\prime}\right]^{-1}$ is homotopic to a certain class in the fiber over $a_{i}$. The image of this class under the translation homomorphism along $l_{i}^{-1}$ is the slope; it is well defined up to a number of moves, irrelevant in the sequel. For details and further properties, see [10].

Now, the monodromy of $C$ is defined as the homomorphism $\mathfrak{m}: \gamma_{i} \mapsto \mathfrak{m}_{i}$, where $\mathfrak{m}_{i}$ is the map $\alpha \mapsto \varkappa_{i}^{-1} \mathfrak{m}_{i}^{\prime}(\alpha) \varkappa_{i}$ and $\mathfrak{m}_{i}^{\prime}=\mathfrak{m}^{\prime}\left(\gamma_{i}\right)$. This monodromy takes values in the extended group $\mathbb{B}_{3} \cdot \operatorname{Inn} \mathfrak{F}$; its image $\mathfrak{I m}_{C}$ is called the monodromy group of $C$. Strictly speaking, both $\mathfrak{m}$ and $\mathfrak{I m}_{C}$ depend on a number of choices (the trigonal model $C^{\prime}$, geometric basis $\left\{\gamma_{i}\right\}$, slopes $\varkappa_{i}$, etc.); however, we only retain the original curve $C$ in the notation as the other choices do not affect the fundamental group. See Theorem 3.4 below.

The projections $\operatorname{pr}_{\Gamma} \mathfrak{I m}_{C}$ and $\operatorname{pr}_{\Gamma} \mathfrak{I m}_{C^{\prime}}$ coincide, hence $\mathfrak{I m}_{C}$ is also a subgroup of genus zero, see Theorem 3.2. Unlike the case of genuine trigonal curves, I do not know an intrinsic description of the subgroups of $\mathbb{B}_{3} \cdot \operatorname{Inn} \mathfrak{F}$ that can appear as the monodromy groups of generalized trigonal curves.

Remark 3.3 (Important remark). It is worth emphasizing that the monodromy groups of genuine and generalized trigonal curves lie, respectively, in the braid group $\mathbb{B}_{3}$ and extended group $\mathbb{B}_{3} \cdot \operatorname{Inn} \mathfrak{F}$. Hence, all statements below concerning 
subgroups of $\mathbb{B}_{3} \cdot \operatorname{Inn} \mathfrak{F}$ or $\mathrm{Bu}_{3}$ hold for generalized trigonal curves, whereas those specific to subgroups of $\mathbb{B}_{3}$ hold for genuine curves only. Formally, one can extend the statements concerning subgroups $G \subset \mathbb{B}_{3}$ and extended modules $\overline{\mathcal{V}}_{G}$ to generalized trigonal curves with all slopes of degree divisible by three.

\subsection{The fundamental group}

Consider a generalized trigonal curve $C \subset \Sigma_{d}$ and pick a nonsingular fiber $F_{0}$ of $C$. The affine and projective fundamental groups of $C$ are defined to be $\pi_{C}^{\text {afn }}=$ $\pi_{1}\left(\Sigma_{d} \backslash(C \cup E \cup F)\right)$ and $\pi_{C}^{\text {proj }}=\pi_{1}\left(\Sigma_{d} \backslash(C \cup E)\right)$. The affine group $\pi_{C}^{\text {afn }}$ is an infinite cyclic central extension of $\pi_{C}^{\text {proj }}$. In particular, the commutants of the two groups are canonically isomorphic, hence so are the Alexander modules defined below.

Fix all necessary data (trigonal model, proper section, bases, an identification $\pi_{F}=\mathfrak{F}$, etc., see Subsections 3.2 and 3.3) and let $\mathfrak{I m}_{C}$ be the resulting monodromy group. The following theorem is essentially contained in [22].

Theorem 3.4. One has $\pi_{C}^{\mathrm{afn}}=\mathfrak{F} /\left\langle\beta(\alpha)=\alpha, \beta \in \mathfrak{I m}_{C}, \alpha \in \mathfrak{F}\right\rangle$.

It follows that $\pi_{C}^{\text {afn }}$ depends only on the conjugacy class of $\mathfrak{I m}_{C} \subset \mathbb{B}_{3} \cdot \operatorname{Inn} \mathfrak{F}$. Any presentation of $\pi_{C}^{\text {afn }}$ as in Theorem 3.4 is called geometric. The group inherits from $\mathfrak{F}$ the degree homomorphism $\operatorname{deg}: \pi_{C}^{\text {afn }} \rightarrow \mathbb{Z}$, which does not depend on the choice of a geometric presentation. (The projective group $\pi_{C}^{\text {proj }}$ is the quotient of $\pi_{C}^{\text {afn }}$ by a certain central element of positive degree.)

Denote by $\mathrm{A}_{C}$ the abelianization of the kernel Ker deg. As in Subsection 2.2, the conjugation $t$ by any element $\alpha \in \pi_{C}^{\text {afn }}$ of degree one turns $\mathrm{A}_{C}$ into a module over $\Lambda$; it is called the Alexander module of $C$, and the order $\Delta_{C, p} \in \Lambda \otimes \mathbb{k}_{p}$ of the $\left(\Lambda \otimes \mathbb{k}_{p}\right)$-module $\mathrm{A}_{C} \otimes \mathbb{k}_{p}$, whenever defined, is called the $(\bmod p)$-Alexander polynomial of $C$. In the classical setting, one usually considers $\Delta_{C}:=\Delta_{C, 0}$. As an immediate consequence of Theorem 3.4, one concludes that $\mathrm{A}_{C}=\mathrm{A}_{G}$, where $G=\mathfrak{I m}_{C}$ is the monodromy group. For this reason, and in view of Theorem 3.2, in the rest of the paper we deal mainly with subgroups rather than curves.

Letting $G=\mathfrak{I m}_{C}$, one can also consider the extended module $\overline{\mathrm{A}}_{C}:=\overline{\mathrm{A}}_{G}$, which 'estimates' $\mathrm{A}_{C}$ from above: there is an epimorphism $\overline{\mathrm{A}}_{C} \rightarrow \mathrm{A}_{C}$ (see Remark 2.13). Note however that $\overline{\mathrm{A}}_{C}$ is not an invariant of the fundamental group $\pi_{C}^{\text {afn }}$ alone: examples in Sections 6 and 7 show that $\overline{\mathrm{A}}_{C}$ may be nontrivial even when $\pi_{C}^{\text {afn }}=\mathbb{Z}$.

Remark 3.5 (Important remark). Summarizing, one concludes that any upper bound on the extended module $\overline{\mathcal{V}}_{G}$ of a subgroup $G$ of $\mathrm{Bu}_{3}$ (respectively, $\mathbb{B}_{3}$ ) of genus zero can serve as an upper bound on the conventional module $\mathcal{V}_{C}$ of a generalized (respectively, genuine) trigonal curve $C$. If $G$ is required to be $\mathbb{S}$-transitive, $C$ must be irreducible. Furthermore, according to Theorem 3.2, any finite index subgroup $G \subset \mathbb{B}_{3}$ of genus zero is the monodromy group of a certain genuine trigonal curve. Hence, all existence statements concerning subgroups of $\mathbb{B}_{3}$ do imply the existence of trigonal curves with desired properties. 
Remark 3.6. In view of Theorem 3.2, the isomorphism $\mathrm{A}_{C}=\mathrm{A}_{G}, G=\mathfrak{I m}_{C}$, makes Definitions 2.11-2.14 geometrically meaningful for subgroups of genus zero. To generalize, one could consider 'trigonal curves' in geometrically ruled surfaces $\Sigma \rightarrow B$ over arbitrary, not necessarily rational, bases. However, in this case the presentation of $\pi_{C}^{\text {afn }}$ is not the one given by Theorem 3.4: $\pi_{C}^{\text {afn }}$ is the quotient of the semidirect product $\mathfrak{F} * \mathfrak{I m}_{C} /\left\langle\beta^{-1} \alpha \beta=\beta(\alpha), \beta \in \mathfrak{I m}_{C}, \alpha \in \mathfrak{F}\right\rangle$ by all elliptic and parabolic elements of $\mathfrak{I m}_{C}$. (A subgroup of genus zero is generated by its elliptic and parabolic elements, see (2.7), and one arrives at the statement of Theorem 3.4.) Thus, it is not quite clear whether one should speak about the Alexander module of $\pi_{C}^{\text {afn }}$ itself (which is always large) or that of the kernel of the inclusion epimorphism $\pi_{C}^{\text {afn }} \rightarrow \pi_{1}(\Sigma) \cong \pi_{1}(B)$. Nor is it clear how the universal subgroups should be defined in this situation.

\subsection{Plane curves with deep singularities}

Let $D \subset \mathbb{P}^{2}$ be a plane curve with a distinguished singular point $P$ of multiplicity $\operatorname{deg} D-3$. Blow $P$ up and consider the proper transform $C$ of $D$ : it is a generalized trigonal curve in the Hirzebruch surface $\Sigma_{1}=\mathbb{P}^{2}(P)$, the exceptional section $E \subset \Sigma_{1}$ being the exceptional divisor of the blow-up. The projection $\Sigma_{1} \rightarrow \mathbb{P}^{2}$ establishes a diffeomorphism

$$
\Sigma_{1} \backslash(C \cup E) \stackrel{\cong}{\longrightarrow} \mathbb{P}^{2} \backslash D
$$

hence an isomorphism $\pi_{C}^{\text {proj }}=\pi_{1}\left(\mathbb{P}^{2} \backslash D\right)$ of the fundamental groups. Thus, all restrictions to the Alexander module/polynomial of a generalized trigonal curve, in particular Theorems 1.2 and 1.3, and Addenda 1.4 and 1.5 in the introduction, hold for plane curves as above. For this reason, we do not mention them separately.

\section{Local geometry of the skeleton}

In this section, we describe the local geometry of the skeleton of a finite index subgroup with nontrivial extended Alexander module. The finite index condition is used in Subsection 4.2: we assume that all regions of the skeleton are bounded.

\subsection{Settings}

Fix a subgroup $G \subset \mathrm{Bu}_{3}$ and let $\mathrm{Sk}=\mathrm{Sk}_{G}$ be its skeleton. We assume that the index $[\Gamma: \bar{G}]$ is finite, so that $\mathrm{Sk}$ is a finite ribbon graph.

Fix, further, a field $\mathbb{k}=\mathbb{k}_{p}$ and an element $\xi$ algebraic over $\mathbb{k}$. Let $\mathbb{K}=\mathbb{k}(\xi)$. Unless stated otherwise, we assume that $\xi \neq \pm 1$. In the rest of the paper, $M$ and $N$ stand for the multiplicative orders of $\xi$ and $-\xi$, respectively. In particular we show that they are finite.

In Subsections 4.2-4.4 below, we pick a vertex $v$ and an edge $e$ close to $v$, define a certain subgroup $G_{v} \subset G$ generated by some loops in a neighborhood of $v$, and consider the submodule $\overline{\mathcal{V}}_{v}(\xi):=\overline{\mathcal{V}}_{G_{v}}(\xi) \subset \mathrm{A}(\xi)$ and the quotient $\overline{\mathrm{A}}_{v}(\xi):=$ 
$\mathrm{A}(\xi) / \overline{\mathcal{V}}_{v}(\xi)$. Then we introduce a basis $\left\{\alpha_{1}, \alpha_{2}, \alpha_{3}\right\}$ over $e$, see Subsection 2.4.5, and use this basis to analyze the conditions, 'local' at $v$, necessary for the nonvanishing $\overline{\mathrm{A}}_{v}(\xi) \neq 0$; this last is equivalent to the requirement that $\operatorname{dim}_{\mathbb{K}} \overline{\mathcal{V}}_{v}(\xi) \leqslant 1$ and is obviously necessary for the nonvanishing $\overline{\mathrm{A}}_{G}(\xi) \neq 0$.

\subsection{A trivalent $\bullet-v e r t e x$}

Consider a trivalent $\bullet$-vertex $v$ of Sk; fix a marking $e$ at $v$ and a corresponding canonical basis $\left\{\alpha_{1}, \alpha_{2}, \alpha_{3}\right\}$. Let $G_{v} \subset G$ be the subgroup generated by the boundaries of $((e))$ and $\left(\left(\mathbb{X}^{-1} e\right)\right)$, i.e., by $t^{r} \sigma_{1}^{m}$ and $t^{s} \sigma_{2}^{n}$, where $m, n>0$ are the widths of the two regions and $r, s$ are given by the corresponding type specifications.

Consider the matrix $\mathcal{M}=\left[t^{r} \sigma_{1}^{m}-\mathrm{id} \mid t^{s} \sigma_{2}^{n}-\mathrm{id}\right]$ :

$$
\mathcal{M}=\left[\begin{array}{cccc}
t^{r}(-t)^{m}-1 & t^{r} \tilde{\varphi}_{m}(-t) & t^{s}-1 & 0 \\
0 & t^{r}-1 & t^{s+1} \tilde{\varphi}_{n}(-t) & t^{s}(-t)^{n}-1
\end{array}\right] .
$$

Clearly, $\operatorname{dim}_{\mathbb{K}} \overline{\mathcal{V}}_{v}(\xi)=\operatorname{rk} \mathcal{M}(\xi)$, and we are interested in the conditions on $m, n, r, s$ necessary and sufficient for $\operatorname{rk} \mathcal{M}(\xi) \leqslant 1$. Consider the following cases.

4.2.1. Type 0. If $\mathcal{M}(\xi)=0$, i.e., $\overline{\mathcal{V}}_{v}(\xi)=0$, the marked vertex $v$ is said to be of type 0 . This is the case if and only if $N:=\operatorname{ord}(-\xi)<\infty$ divides both $m$ and $n$ and $\mathrm{e}_{p}(N)$ divides both $r$ and $s$.

Now, assume that $\overline{\mathcal{V}}_{v}(\xi) \neq 0$ is a proper submodule of $\mathrm{A}(\xi)$. Then $\xi$ annihilates all $(2 \times 2)$-minors of $\mathcal{M}$ and one has one of the following three cases.

4.2.2. Type $\mathbf{I}_{\mathbf{1}} \cdot \xi^{r}(-\xi)^{m}-1=\tilde{\varphi}_{m}(-\xi)=\xi^{s}-1=0$, i.e., the first row vanishes. In this case, $N:=\operatorname{ord}(-\xi) \mid m$; in addition, one has $\xi^{r}=\xi^{s}=1$, i.e., $\mathrm{e}_{p}(N)$ divides both $r$ and $s$. The module $\overline{\mathcal{V}}_{v}(\xi)$ is generated by $\tilde{\varphi}_{n}(-t) \mathbf{e}_{2}$. If $N \nmid n$, the marked vertex $v$ is said to be of type $\mathrm{I}_{1}$. Then $\overline{\mathcal{V}}_{v}(\xi) \neq 0$ is generated by $\mathbf{e}_{2}$.

4.2.3. Type $\mathbf{I}_{2} \cdot \xi^{r}-1=\tilde{\varphi}_{n}(-\xi)=\xi^{s}(-\xi)^{n}-1=0$, i.e., the second row vanishes. Similarly to the previous case, $N:=\operatorname{ord}(-\xi) \mid n$ and $\mathrm{e}_{p}(N)$ divides both $r$ and $s$. The module $\overline{\mathcal{V}}_{v}(\xi)$ is generated by $\tilde{\varphi}_{m}(-t) \mathbf{e}_{1}$. If $N \nmid m$, the marked vertex $v$ is said to be of type $\mathrm{I}_{2}$. In this case, $\overline{\mathcal{V}}_{v}(\xi) \neq 0$ is generated by $\mathbf{e}_{1}$.

4.2.4. Type II. $\xi^{r}(-\xi)^{m}-1=\xi^{s}(-\xi)^{n}-1=\mathcal{M}_{2,3}(\xi)=0$, where $\mathcal{M}_{2,3}$ is the minor composed of the second and third columns. Modulo the first two relations, $\xi^{r} \tilde{\varphi}_{m}(-\xi)=\left(\xi^{r}-1\right) /(\xi+1)$ and $\xi^{s+1} \tilde{\varphi}_{n}(-\xi)=\xi\left(\xi^{s}-1\right) /(\xi+1)$, see $(2.2)$, and $\mathcal{M}_{2,3}(\xi)=-\left(\xi^{r}-1\right)\left(\xi^{s}-1\right)\left(\xi^{2}+\xi+1\right) /(\xi+1)^{2}$. Thus, either

1. $\xi^{r}=1$, and then $N:=\operatorname{ord}(-\xi) \mid m$, or

2. $\xi^{s}=1$, and then $N:=\operatorname{ord}(-\xi) \mid n$, or

3. $\xi^{2}+\xi+1=0$.

Using (2.2) and the fact that $t+1$ is invertible in $\Lambda(\xi)$, one can see that the module $\overline{\mathcal{V}}_{v}(\xi)$ is generated by

$$
\left(t^{s}-1\right)\left(\left(t^{-1}+1\right) \mathbf{e}_{1}+\mathbf{e}_{2}\right) \text { and }\left(t^{r}-1\right)\left(\mathbf{e}_{1}+(t+1) \mathbf{e}_{2}\right)
$$


in Cases (1) and (2), respectively. In Case (1), assuming that $\xi^{s} \neq 1$ (and hence $N \nmid n)$, the vertex is said to be of type $\mathrm{II}_{1}$; the module $\overline{\mathcal{V}}_{v}(\xi)$ is generated by $\left(t^{-1}+1\right) \mathbf{e}_{1}+\mathbf{e}_{2}$. In Case (2), assuming that $\xi^{r} \neq 1$ (and hence $N \nmid m$ ), the vertex is said to be of type $\mathrm{II}_{2}$; the module $\overline{\mathcal{V}}_{v}(\xi)$ is generated by $\mathbf{e}_{1}+(t+1) \mathbf{e}_{2}$.

In Case (3), assuming that $\xi^{r} \neq 1$ and $\xi^{s} \neq 1$, we let $N=\mathrm{e}_{p}(3)$ and assign to the vertex type $\mathrm{II}_{\mathrm{ex}}$. This is the only case when one cannot assert that $N \mid m$ or $N \mid n$. (In fact, if $N$ does divide $m$ or $n$, then the vertex is of type $0, \mathrm{II}_{1}$, or $\mathrm{II}_{2}$.) The module $\overline{\mathcal{V}}_{v}(\xi)$ is generated by any of the two elements $\left(t^{-1}+1\right) \mathbf{e}_{1}+\mathbf{e}_{2}$ or $\mathbf{e}_{1}+(t+1) \mathbf{e}_{2}$ above.

Summarizing, one concludes that a necessary condition for the nonvanishing $\mathrm{A}(\xi) / \overline{\mathcal{V}}_{G}(\xi) \neq 0$ is that $N:=\operatorname{ord}(-\xi)<\infty$ and at each marked vertex $(v, e)$ other than of type $\mathrm{II}_{\mathrm{ex}}$ (which can only occur if $\mathrm{e}_{p}(N)=3$ ) at least one of the regions $((e)),\left(\left(\mathbb{X}^{-1} e\right)\right)$ has width divisible by $N$.

Definition 4.2. With $N$ fixed, a region of width divisible by $N$ is called trivial (or $N$-trivial); such a region does not contribute to $\overline{\mathcal{V}}_{G}(\xi)$. A region of width not divisible by $N$ is called essential, or $N$-essential. Essential regions are subdivided into type I and II, depending on the types of the vertices in their boundaries.

Summarizing, one arrives at the following statement.

Lemma 4.3. Assume that $\overline{\mathrm{A}}_{G}(\xi) \neq 0$ and let $M=\mathrm{e}_{p}(N)$. Then:

1. for each trivial region $R$ one has $\operatorname{tp}(R)=\operatorname{wd} R \bmod 2 M$;

2. for each type I essential region $R$ one has $\operatorname{tp}(R)=\operatorname{wd} R \bmod 2 M$;

3. for a type II essential region $R$ of width $n=\operatorname{wd} R$, if $n$ is even or $p=2$, then $\operatorname{tp}(R)=-n \bmod 2 M$, otherwise $\operatorname{tp}(R)=M-n \bmod 2 M$, and in the latter case $M$ must be even;

4. if $M \neq 3$, at each trivalent $\bullet$-vertex at most one region is essential;

5. if $\overline{\mathcal{V}}_{G}(\xi)=0$, then all regions are trivial.

Proof. Items (1)-(3) paraphrase the conditions $t^{s}=1$ and $t^{s}(-1)^{n}=1$ in terms of the type specification. For (4) and (5), it suffices to consider all three markings at the given vertex or, respectively, at all vertices of the skeleton.

\subsection{A monovalent $\bullet-v e r t e x($ type III)}

Consider a monovalent •-vertex $v$ and let $e$ be the adjacent edge. In a canonical basis $\left\{\alpha_{1}, \alpha_{2}, \alpha_{3}\right\}$ over $e$, the positive loop about $v$ lifts to an element of the form $t^{r}\left(\sigma_{2} \sigma_{1}\right)$. Let $G_{v} \subset G$ be the subgroup generated by this element.

One has $\operatorname{det}\left(t^{r}\left(\sigma_{2} \sigma_{1}\right)-\mathrm{id}\right)=\tilde{\varphi}_{3}\left(t^{r+1}\right)$. Hence, one has $\overline{\mathrm{A}}_{v}(\xi) \neq 0$ if and only if $M:=\operatorname{ord} \xi<\infty$ satisfies the following conditions:

- $M \mid 3(r+1)$ and $M \nmid(r+1)$ (in particular, $M=0 \bmod 3)$ if $p \neq 3$, and

- $M \mid(r+1)$ and $M \neq 0 \bmod 3$ if $p=3$.

If this is the case, the module $\overline{\mathcal{V}}_{v}(\xi)$ is generated by $-t^{r} \mathbf{e}_{1}+\mathbf{e}_{2}$. Computing the exponents modulo $M$, the latter can be rewritten in the form $-t^{s} \mathbf{e}_{1}+\mathbf{e}_{2}$, where 
- $s= \pm \frac{1}{3} M-1$ if $p \neq 3$ and

- $s=-1$ (or $s=M-1$ ) if $p=3$.

If $p \neq 3$ then, according to the sign \pm in the expression for $s$ above, we assign to the vertex $v$ type $\mathrm{III}_{ \pm}$. If $p=3$, there is one type III. Observe that, if $p \neq 3$, the generator of $\overline{\mathcal{V}}_{G}(\xi)$ can be rewritten in the form $-\epsilon t^{-1} \mathbf{e}_{1}+\mathbf{e}_{2}$ with $\epsilon^{2}+\epsilon+1=0$.

Now, assume that $v$ has a trivalent neighbor $u$ in Sk. (The remaining cases are treated in Subsection 4.5 below.) Summarizing and using Lemma 4.3, one arrives at the following statement.

Lemma 4.4. Assume that $\overline{\mathrm{A}}_{G}(\xi) \neq 0$ and let $M=\mathrm{e}_{p}(N)$. Let $v$ be a monovalent -vertex and let $u$ be its trivalent neighbor. Then:

1. if $p \neq 3$, then $M=0 \bmod 3$ and $\operatorname{tp}(v)= \pm \frac{2}{3} M \bmod 2 M$;

2. if $p=3$, then $M \neq 0 \bmod 3$ and $\operatorname{tp}(v)=0 \bmod 2 M$;

3. unless $u$ is of type $\mathrm{II}_{\mathrm{ex}}, v$ is in the boundary of an $N$-trivial region.

Remark 4.5. If $G \in \mathbb{B}_{3}$ and $p \neq 3$, see Lemma $4.4(1)$, the condition $\operatorname{tp}(\bullet)=2$ $\bmod 6$ in Proposition 2.9 (3) implies that $M= \pm 3 \bmod 9$ and, according to the sign in this congruence, only one type $\mathrm{III}_{ \pm}$can appear.

\subsection{A monovalent o-vertex (type IV)}

Consider a monovalent o-vertex $v$ and let $e^{\prime}$ be the adjacent edge. To simplify the expressions below, switch to the edge $e=\mathbb{X} \mathbb{Y} e^{\prime}$. In a canonical basis $\left\{\alpha_{1}, \alpha_{2}, \alpha_{3}\right\}$ over $e$, the positive loop about $v$ lifts to an element of the form $t^{r}\left(\sigma_{2} \sigma_{1} \sigma_{2}\right)$. Let $G_{v} \subset G$ be the subgroup generated by this element.

One has $\operatorname{det}\left(t^{r}\left(\sigma_{2} \sigma_{1} \sigma_{2}\right)-\mathrm{id}\right)=1-t^{2 r+3}$. Hence, one has $\overline{\mathrm{A}}_{v}(\xi) \neq 0$ if and only if $M:=\operatorname{ord} \xi \mid(2 r+3)$, and in this case $\overline{\mathcal{V}}_{v}(\xi)$ is generated by $t^{r+1} \mathbf{e}_{1}+\mathbf{e}_{2}$, which can be rewritten in the form $t^{s} \mathbf{e}_{1}+\mathbf{e}_{2}$, where $s=\frac{1}{2}(M-1)$.

A monovalent o-vertex $v$ is said to be of type IV. Assuming that $v$ is adjacent to a trivalent vertex $u$, one arrives at the following statement.

Lemma 4.6. Assume that $\overline{\mathrm{A}}_{G}(\xi) \neq 0$ and let $M=\mathrm{e}_{p}(N)$. Let $v$ be a monovalent o-vertex and $u$ its trivalent neighbor. Then:

1. $M$ is odd and $\operatorname{tp}(v)=M \bmod 2 M$;

2. unless $u$ is of type $\mathrm{II}_{\mathrm{ex}}, v$ is in the boundary of an $N$-trivial region.

\subsection{Two special subgroups}

In this subsection, we treat the two cases that are not quite covered by Lemmas 4.4 and 4.6; namely, we consider a skeleton Sk with a monovalent •- or o-vertex that is not adjacent to a trivalent $\bullet$-vertex. Clearly, Sk is either $\circ \bullet$ or $\bullet \bullet$; in the former case, $\bar{G}=\Gamma$, in the latter case, $\bar{G}$ is the only index 2 subgroup $\Gamma^{2}=2 A^{0}$.

Proposition 4.7. If $\bar{G}=\Gamma$, then $\mathrm{A}_{G}(\xi) \neq 0$ if and only if $G \prec(\Gamma)^{-}$. In this case, one has $p=2, \xi^{2}+\xi+1=0$, and $\overline{\mathcal{V}}_{G}(\xi)=\mathbb{K}\left(-t \mathbf{e}_{1}+\mathbf{e}_{2}\right)$.

Proof. It suffices to consider the matrix $\mathcal{M}$ in (4.1) with $m=n=1$. 
Proposition 4.8. Assume that $\bar{G}=\Gamma^{2}=2 A^{0}$ and $\mathrm{A}_{G}(\xi) \neq 0$. Then either

1. $p=3, \xi=1$, and $\overline{\mathcal{V}}_{G}(\xi)=\mathbb{K}\left(-t \mathbf{e}_{1}+\mathbf{e}_{2}\right)$; in which case $G \prec\left(2 A^{0}\right)^{\text {bu }}$, or

2. $\xi^{2}+\xi+1=0$ and $\overline{\mathcal{V}}_{G}=\Lambda\left(-t \mathbf{e}_{1}+\mathbf{e}_{2}\right) \bmod \Phi_{3}$; in which case $G \prec\left(2 A^{0}\right)^{-}$.

Proof. The group is generated by $t^{r} \sigma_{1}^{2}, t^{s} \sigma_{2}^{2}, t^{k} \sigma_{2} \sigma_{1}$, and, possibly, an extra power of $t$, and the proof is a direct computation, starting with (4.1) with $m=n=2$; see Subsections 4.2 and 4.3 .

Remark 4.9. The largest subgroup of $\Gamma$ (respectively, $\Gamma^{2}=2 A^{0}$ ) on which the slope $-\mathrm{dg}$ is equal to $\operatorname{dg} \bmod 6$ is $\Gamma(3)=3 D^{0}=\operatorname{Ker}(\operatorname{dg} \bmod 3)$ (respectively, $\Gamma^{\prime}=6 A^{1}=\operatorname{Ker}(\operatorname{dg} \bmod 6)$; this latter subgroup is of genus one $)$.

\subsection{A few consequences}

We state a few immediate consequences of the computation in Subsections 4.2-4.4. Note that in Lemma 4.10 we do not assume that $\operatorname{dp} G \neq 0$ (which would make the claim trivial).

Lemma 4.10. If $\bar{G} \subset \Gamma$ is a subgroup of finite index, there is an integer $M>0$ such that $\left(t^{M}-1\right)\left(\mathrm{A}_{G} \otimes \mathbb{k}_{p}\right)=0$ for each $p$. In particular, the Alexander polynomial $\bar{\Delta}_{G, p}$ is well defined and divides $\left(t^{M}-1\right)^{2}$.

Proof. One merely repeats the arguments of Subsections 4.2 and 4.5, computing the ranks of the corresponding matrices over $\Lambda \otimes \mathbb{k}_{p}$. Each time the rank is 2 and all invariant factors divide some $\left(t^{M}-1\right)$.

Lemma 4.11. If $\overline{\mathcal{V}}_{G}(\xi)=0$, i.e., if $\mathrm{rk}_{G}=2$, then all vertices of $\mathrm{Sk}_{G}$ are trivalent (equivalently, $G$ is torsion free) and all regions of $\mathrm{Sk}_{G}$ are trivial.

Proof. According to Subsections 4.2, 4.3, and 4.4, each essential region of $\mathrm{Sk}_{G}$ and each monovalent vertex makes a nontrivial contribution to $\overline{\mathcal{V}}_{G}(\xi)$.

\subsection{Isotrivial curves}

Recall that, in appropriate affine coordinates $(x, y)$ on $\Sigma_{d}$, the equation of an irreducible isotrivial genuine trigonal curve $C$ can be written in the form

$$
y^{2}=\prod_{i}\left(x-x_{i}\right)^{m_{i}}, \quad m:=\text { g.c.d. }\left(m_{i}\right) \neq 0 \bmod 3 .
$$

Hence, the monodromy group of any generalized trigonal curve Nagata equivalent to $C$ is the abelian group generated by $t^{r}\left(\sigma_{2} \sigma_{1}\right)$ and $t^{s}$ id for some $r, s \in \mathbb{Z}$.

Theorem 4.12. The extended Alexander polynomial of an irreducible isotrivial generalized trigonal curve $C$ divides $\tilde{\varphi}_{3}\left(t^{r+1}\right)$ for some $r \in \mathbb{Z}$. If $C$ is a genuine curve, then $\bar{\Delta}_{C, p}=\tilde{\varphi}_{3}\left(t^{r+1}\right)$ for some $r \in 3 \mathbb{Z}$ and any $p$.

Proof. Both statements follow from the description of the monodromy group and the computation in Subsection 4.3. If $C$ is a genuine curve, the monodromy group is generated by $\left(\sigma_{2} \sigma_{1}\right)^{m}$, hence $r \in 3 \mathbb{Z}$ and $s=0$. 


\section{Proof of Theorem 1.3}

Throughout this section, we fix $p$ (a prime or zero), a subgroup $G \subset \mathrm{Bu}_{3}$ of genus zero, and a root $\xi$ of its Alexander polynomial $\bar{\Delta}_{G, p}$. Let $N=\operatorname{ord}(-\xi)<\infty$. Recall that we assume $\xi \neq \pm 1$, hence $N \geqslant 3$. The ultimate goal of the section is a proof of Theorem 1.3 and the estimate $N \leqslant 10$ for $p=0$, see Corollary 5.10.

\subsection{The boundary of a trivial region}

Consider an $N$-trivial region $R$ of a certain width $N m$. With respect to the default marking, see Subsection 2.4.3, all vertices in $\partial R$ are of types $0, \mathrm{I}_{1}, \mathrm{II}_{1}, \mathrm{III}_{ \pm}$(or III if $p=3$ ), or IV. Define the distance $\operatorname{dist}\left(v_{1}, v_{2}\right) \in \mathbb{Z}_{N m}$ between two boundary vertices $v_{1}, v_{2} \in \partial R$ as the distance in $R$, regarded as an orbit of $\mathbb{X} \mathbb{Y}$, between the corresponding edges $e_{1}, e_{2}$ used in Subsections 4.2-4.4 to construct the canonical bases.

Lemma 5.1. With two exceptions, the distance in $\partial R$ between any two vertices of the same type other than 0 is divisible by $N$. The exceptions are as follows:

- $\mathrm{e}_{p}(N)=3$ and the vertices are of type II or III $_{-}$, or

- $\mathrm{e}_{p}(N)=3, p=2$, and the vertices are of type IV.

Proof. Let $M=\mathrm{e}_{p}(N)$. Consider a vertex $v \in \partial R$ of a type other than $0, \mathrm{I}_{2}$, or $\mathrm{II}_{2}$ (the two latter do not occur due to our choice of the markings). According to Subsections $4.2-4.4$, in the corresponding canonical basis the submodule $\overline{\mathcal{V}}_{v}(\xi)$ is generated by a vector of the form $a_{v}(t) \mathbf{e}_{1}+\mathbf{e}_{2}$, where the coefficient $a_{v}(t) \in \Lambda(\xi)$ depends on $\xi$ and the type of $v$ only. One has

$$
a_{v}(t)=0, \quad t^{-1}+1, \quad-t^{s}, \quad \text { or } \quad t^{\frac{1}{2}(M-1)}
$$

for $v$ of type $\mathrm{I}_{1}, \mathrm{II}_{1}, \mathrm{III}_{ \pm}$(or III if $p=3$ ), or IV, respectively. Here, $s= \pm \frac{1}{3} M-1$ for type $\mathrm{III}_{ \pm}$and $s=-1$ for type III.

Let $u \subset \partial R$ be another vertex at a distance $d$ from $v$. Connecting the corresponding edges by a path in $\partial R$, one can assume that the canonical bases used are related via $\sigma_{1}^{d}$, and a necessary condition for $\overline{\mathrm{A}}_{G}(\xi) \neq 0$ is that the generators of $\overline{\mathcal{V}}_{u}(\xi)$ and $\sigma_{1}^{d} \overline{\mathcal{V}}_{v}(\xi)$ should be linearly dependent. This condition results in the equation

$$
\tilde{\varphi}_{d}(-\xi)\left((\xi+1) a_{v}(\xi)-1\right)=a_{v}(\xi)-a_{u}(\xi)
$$

If $u$ and $v$ are of the same type, the right hand side vanishes and (5.3) takes the form $\tilde{\varphi}_{d}(-\xi)=0$ or $(\xi+1) a_{v}(\xi)=1$. In the former case, one has $N \mid d$, as stated; in the latter case, using the list above, one can see that the equation has no solutions (for type $\mathrm{I}_{1}$ ), implies $\xi=1$ (for type III with $p=3$ ), or implies $\xi^{2}+\xi+1=0$. Indeed, if $v$ is a vertex of type $\mathrm{II}_{1}$, the equation $(\xi+1) a_{v}(\xi)=1$ is equivalent to $\xi^{2}+\xi+1=0$. If $v$ is of type $\mathrm{III}_{ \pm}$, then, switching to $a_{v}(t)=-\epsilon t^{-1}$ with $\epsilon^{2}+\epsilon+1=0$, see Subsection 4.3 , one has $\xi=-\epsilon /(\epsilon+1)$, hence $\xi^{2}+\xi+1=0$. 
Furthermore, in this case $\epsilon \xi^{-1}=\xi$, i.e., the type is III_. If $v$ is of type IV, then, letting $s=\frac{1}{2}(M-1)$ and hence $M=2 s+1$, one has

$$
\left[t^{s}(t+1)-1\right] \cdot t\left[t^{s}(t+1)+1\right]-\left[t^{2 s+1}-1\right] \cdot(t+1)^{2}=t^{2}+t+1
$$

Then $M=3$ and the equation turns into $\xi^{2}+\xi=1$. Hence $p=2$.

Lemma 5.4. If $p=0$ and $N \neq 4$, the boundary $\partial R$ cannot contain vertices of types both $\mathrm{I}_{1}$ and $\mathrm{II}_{1}$.

Proof. Assume that $v$ is of type $\mathrm{I}_{1}$ and $u$ is of type $\mathrm{II}_{1}$. Then (5.3) turns into the four term equation $\xi(-\xi)^{d}+\xi^{2}+\xi+1=0$, in which each term is a root of unity. Geometrically, the sum of four unit complex numbers equals zero if and only if the summands split into two pairs of opposite ones. Hence, the above equation implies $\xi=-1$ or $\xi= \pm i$; in the latter case, one has $N=4$.

\subsection{First estimates}

Denote by $R_{i}$ and $S_{j}$, respectively, the trivial and essential regions of Sk (where $i$ and $j$ run over certain index sets). Introduce the following counts for Sk:

- $v_{1}$ is the number of monovalent $\bullet$-vertices;

- $v_{3}$ is the number of trivalent $\bullet$-vertices;

- $e_{1}$ is the number of monovalent o-vertices;

- $e_{2}$ is the number of edges connecting pairs of $\bullet$-vertices;

- $N m_{i}$ is the width of the trivial region $R_{i}$; let $m=\sum_{i} m_{i}$;

- $n_{j}$ is the width of the essential region $S_{j}$; let $n=\sum_{j} n_{j}$.

For a trivial region $R_{i}$, introduce also the following parameters, counting special vertices in the boundary $\partial R_{i}$ :

- $K_{i}^{\mathrm{I}}$ is the number of vertices of type $\mathrm{I}_{1}$ or $\mathrm{II}_{1}$;

- $K_{i}^{\mathrm{III}}$ is the number of vertices of type $\mathrm{III}_{ \pm}$(or III if $p=3$ );

- $K_{i}^{\mathrm{IV}}$ is the number of vertices of type IV.

For $*=\mathrm{I}$, III, or IV, let $k_{i}^{*}=K_{i}^{*} / m_{i}$ and $k^{*}=\max _{i} k_{i}^{*}$. Unless $\mathrm{e}_{p}(N)=3$, in view of Lemma 5.1 , one has $0 \leqslant k^{\mathrm{I}}, k^{\mathrm{III}} \leqslant 2$ and $0 \leqslant k^{\mathrm{IV}} \leqslant 1$ and, due to Lemma 5.4 , one has $k^{\mathrm{I}} \leqslant 1$ if $p=0$ and $N \neq 4$. Furthermore, $k^{\mathrm{III}} \leqslant 1$ if $p=3$ and $k^{\mathrm{III}}$ and $k^{\mathrm{IV}}$ vanish unless $e_{p}(N)$ satisfies certain divisibility conditions, see Lemmas 4.4 and 4.6 for the existence of vertices of the corresponding types.

The total number of regions of Sk does not exceed $m+n$ and, since Sk is a ribbon graph of genus zero, Euler's theorem implies $m+n-e_{2}+v_{1}+v_{3} \geqslant 2$. (The edges counted by $e_{1}$ are cancelled by the monovalent o-vertices.) As usual, one has $v_{1}+3 v_{3}=N m+n=e_{1}+2 e_{2}$, and, eliminating $e_{2}$ and $v_{3}$, one can rewrite Euler's inequality above in the form

$$
(6-N) m+5 n+4 v_{1}+3 e_{1} \geqslant 12 .
$$


Since all monovalent vertices belong to the boundaries of trivial regions of Sk, see Lemmas 4.4 and 4.6, one has

$$
v_{1}=\sum_{i} k_{i}^{\mathrm{III}} m_{i} \leqslant k^{\mathrm{III}} m, \quad e_{1}=\sum_{i} k_{i}^{\mathrm{IV}} m_{i} \leqslant k^{\mathrm{IV}} m .
$$

The following observation is crucial.

Lemma 5.5. One has $n=\sum_{i} k_{i}^{\mathrm{I}} m_{i} \leqslant k^{\mathrm{I}} m$.

Proof. By definition, each vertex in the boundary of an essential region is of type I or II. On the other hand, due to Lemma 4.3 (4), each such vertex $v$ appears in the boundary of an essential region exactly once (hence the number of these vertices is $n$ ) and admits a unique marking $e$ with respect to which it is of type $\mathrm{I}_{1}$ or $\mathrm{II}_{1}$. With this marking, $((e))$ is a trivial region; hence $v$ is counted exactly once in the $\operatorname{sum} \sum_{i} k_{i}^{\mathrm{I}} m_{i}$.

Substituting, one arrives at

$$
(6-N) m+\sum_{i}\left(5 k_{i}^{\mathrm{I}}+4 k_{i}^{\mathrm{III}}+3 k_{i}^{\mathrm{IV}}\right) m_{i} \geqslant 12
$$

and $\left(6+k^{\text {all }}-N\right) m \geqslant 12$, where $k^{\text {all }}=\max _{i}\left(5 k_{i}^{\mathrm{I}}+4 k_{i}^{\mathrm{III}}+3 k_{i}^{\mathrm{IV}}\right)$. This implies

$$
N<6+k^{\text {all }} \leqslant 6+5 k^{\mathrm{I}}+4 k^{\mathrm{III}}+3 k^{\mathrm{IV}} .
$$

Corollary 5.8. If $p=$ char $\mathbb{k}=0$, then $N \leqslant 21$; otherwise, $N \leqslant 26$.

Proof. The statement follows from (5.7) and the estimates on $k^{\mathrm{I}}, k^{\mathrm{III}}$, and $k^{\mathrm{IV}}$ listed right after their definition.

\subsection{Further restrictions}

We keep the notation introduced in Subsection 5.2.

Lemma 5.9. If $N>10$ and the triple $\left(p, N, \varkappa_{\xi}\right)$ is not one of those listed in Tables 1 and 2 , then, for each trivial region $R_{i}$, one has $k_{i}^{\mathrm{I}}+k_{i}^{\mathrm{III}}+k_{i}^{\mathrm{IV}} \leqslant 1$.

Proof. It suffices to show that, with the stated assumptions, the distance in $\partial R_{i}$ between any two vertices $u$ and $v$ of types, respectively, $T_{u}$ and $T_{v}$ other than 0 is divisible by $N$. Due to Lemma 5.1, one can assume that $T_{u} \neq T_{v}$. Let $d=$ $\operatorname{dist}(u, v)$. Then $\xi$ must satisfy (5.3) and, since the equation is obviously $N$-periodic in $d$, it suffices to consider the values $d=1, \ldots, N-1$.

Now, for each $N=11, \ldots, 26$, see Corollary 5.8 , each $d=1, \ldots, N-1$, and each pair $T_{u} \neq T_{v}$ of types, consider the resultant $\mathcal{R}$ of $(5.3)$ and $(-\xi)^{N}-1$. (All computations below were performed using Maple.) One has $\mathcal{R} \neq 0$, which proves the statement for $p=0$. For each prime divisor $p \neq 2,3$ of $\mathcal{R}$, consider the greatest common divisor of the two polynomials over $\mathbb{F}_{p}$, decompose it into irreducible factors, and select those that do not divide $(-\xi)^{n}-1$ for some $n<N$. The minimal polynomial $\varkappa_{\xi}$ must be one of these factors. The cases $p=2$ or 3 are treated similarly, but separately, as equation (5.3) changes in these cases.

The above procedure results in a finite collection (too large to be listed here) of sequences $\left(N, p, \varkappa_{\xi} ; d, T_{u}, T_{v}\right)$. For each triple $\left(N, p, \varkappa_{\xi}\right)$ thus obtained, one can 
TABLE 2. Exceptional factors of $\Delta$ (not realized)

\begin{tabular}{rrl}
\multicolumn{1}{c}{$p$} & $\mathrm{~N}$ & \multicolumn{1}{c}{ Factors $\varkappa_{\xi} \in \mathbb{F}_{p}[t]$ of $\Delta$} \\
\hline 3 & 13 & $t^{3}+2 t+1, t^{3}+2 t^{2}+1, t^{3}+t^{2}+2 t+1, t^{3}+2 t^{2}+t+1$ \\
23 & 11 & $t+2, t+4, t+6, t+9, t+12, t+18$ \\
29 & 14 & $t+4, t+22$ \\
31 & 15 & $t+14, t+18, t+19, t+20$ \\
37 & 12 & $t+8, t+14, t+23, t+29$ \\
43 & 14 & $t+32, t+39$ \\
& 21 & $t+14, t+40$ \\
53 & 13 & $t+28, t+36$ \\
61 & 15 & $t+16, t+42$ \\
79 & 13 & $t+38, t+52$ \\
127 & 21 & $t+47, t+100$ \\
211 & 15 & $t+83, t+150$
\end{tabular}

analyze the types of vertices that may appear simultaneously in the boundary of a single region and improve the a priori estimate $k^{\text {all }} \leqslant 21$ used in (5.7). (For example, if all types that can appear in the same region are I, II, and $\mathrm{III}_{+}$, the estimate improves to $k^{\text {all }} \leqslant 14$, hence $N \leqslant 19$.) Disregarding the triples that do not satisfy the new inequality $N<6+k^{\text {all }}$, one obtains Tables 1 and 2 .

Corollary 5.10. Unless $\left(p, N, \varkappa_{\xi}\right)$ is one of the triples listed in Tables 1 and 2, one has $N \leqslant 10$.

Proof. Replacing all coefficients in the definition of $k^{\text {all }}$ with their maximum 5 and using Lemma 5.9, one obtains $k^{\text {all }} \leqslant 5$ in (5.7).

\subsection{Proof of Theorem 1.3 and Addendum 1.5}

Due to Remark 3.5, it suffices to prove a similar statement for the extended Alexander modules $\overline{\mathcal{V}}_{G}$ of subgroups $G \subset \mathrm{Bu}_{3}$ of genus zero. Note that we do not use S-transitivity.

Consider one of the triples $\left(p, N, \varkappa_{\xi}\right)$ listed in Tables 1 and 2. The submodule $\overline{\mathcal{V}}_{G}(\xi)$ has the form $\mathbb{K} \mathbf{v}$, where $\mathbf{v}$ is one of the vectors listed in (5.2). We choose $\mathbf{v}=\mathbf{e}_{2}$ and compute the genus of the corresponding universal subgroup. The computation, using Maple, proceeds as follows. Map $\mathbb{B}_{3}$ or $\mathrm{Bu}_{3}$ to the finite group $G L(2, \mathbb{K})$, let $\mathcal{V}=\mathbb{K} \mathbf{e}_{2}$, and enumerate the cosets modulo the universal subgroup $G_{\mathcal{V}}$. In order to pass to $\bar{G}_{\mathcal{V}}$, identify further $\mathcal{M}$ and $t^{s} \mathcal{M}$ for $\mathcal{M} \in \operatorname{Mat}_{2 \times 2}(\mathbb{K})$ and $s \in \mathbb{Z}_{M}$. (If a subgroup of $\mathbb{B}_{3}$ is to be found, take only $s \in 3 \mathbb{Z}_{M}$.) The result is the set of edges of the skeleton of $G_{\mathcal{V}}$, see Subsection 2.4, its •- and o-vertices and regions being the orbits of $\sigma_{2} \sigma_{1}, \sigma_{2} \sigma_{1}^{2}$, and $\sigma_{1}$, respectively. Compute the Euler characteristic and make sure that it equals 2 .

After the computation is completed, one can use the cosets found to verify that, in fact, all the subspaces $\mathbb{K} \mathbf{v}$ with $\mathbf{v}$ as in (5.2) are conjugate to $\mathbb{K} \mathbf{e}_{2}$; hence they would yield the same universal subgroups. 
TABLE 3 . Examples with $7 \leqslant N \leqslant 10$

\begin{tabular}{rrll}
\multicolumn{1}{c}{$p$} & $\mathrm{~N}$ & \multicolumn{1}{c}{ Factors $\varkappa_{\xi} \in \mathbb{k}[t]$ of $\Delta$} & $\bar{G} \subset \Gamma$ \\
\hline 2 & $* 7$ & $t^{3}+t+1, t^{3}+t^{2}+1$ & $\left(9 ; 1,0 ; 1^{2} 7^{1}\right)$ \\
3 & $* 8$ & $t^{2}+2 t+2, t^{2}+t+2$ & $\left(10 ; 0,1 ; 1^{2} 8^{1}\right)$ \\
5 & $* 8$ & $t^{2}+2, t^{2}+3$ & $\left(78 ; 0,0 ; 1^{6} 8^{9}\right)$ \\
11 & $* 10$ & $t+2, t+6, t+7, t+8$ & $\left(24 ; 2,0 ; 1^{2} 2^{1} 10^{2}\right)$ \\
17 & $* 8$ & $t+2, t+8, t+9, t+15$ & $\left(36 ; 0,0 ; 1^{4} 8^{4}\right)$ \\
19 & 9 & $t+4, t+5, t+6, t+16, t+9, t+17$ & $\left(20 ; 0,2 ; 1^{2} 9^{2}\right)$ \\
29 & $* 7$ & $t+7, t+16, t+20, t+23, t+24, t+25$ & $\left(60 ; 0,0 ; 1^{4} 7^{8}\right)$ \\
37 & 9 & $t+7, t+9, t+12, t+16, t+33, t+34$ & $\left(76 ; 0,4 ; 1^{4} 9^{8}\right)$ \\
43 & $* 7$ & $t+4, t+11, t+16, t+21, t+35, t+41$ & $\left(132 ; 0,0 ; 1^{6} 7^{18}\right)$
\end{tabular}

This computation eliminates all the triples listed in Table 2 and, for subgroups of $\mathbb{B}_{3}$, the values $p=5$ and 19 in Table 1 , thus completing the proof of Theorem 1.3 and the existence part of Addendum 1.5. (For the existence, one should also use Remark 3.5 and, passing from $\overline{\mathcal{V}}_{G}$ to $\mathcal{V}_{G}$, Corollary 2.18.)

Further analysis of the data obtained in Lemma 5.9 shows that, with $N$ and $p$ fixed, each triple $\left(d, T_{u}, T_{v}\right)$ gives rise to at most one irreducible factor $\varkappa_{\xi}$. Hence, this factor is uniquely recovered from the geometry of any trivial region of the skeleton containing vertices of more than one type (such a region must exist to break the bound $k^{\text {all }} \leqslant 5$, cf. Corollary 5.10), and two distinct factors cannot appear simultaneously.

Remark 5.11. Since the images of $\mathbb{B}_{3}$ and $\mathrm{Bu}_{3}$ in $G L(2, \mathbb{K})$ are not known, the coset enumeration procedure starts with the identity and keeps multiplying matrices by $\sigma_{2} \sigma_{1}$ and $\sigma_{1} \sigma_{2} \sigma_{1}$, comparing the result with all matrices already listed; each new matrix $\mathcal{M}$ is added to the list together with all products $t^{s} \mathcal{M}, s=1, \ldots, M-1$. (If $M=0 \bmod 3$ and a subgroup of $\mathbb{B}_{3}$ is to be found, only values $s=0 \bmod 3$ are used.) The equivalence relation is linear: two matrices $\mathcal{M}_{1}, \mathcal{M}_{2} \in \operatorname{Mat}_{2 \times 2}(\mathbb{K})$ are equivalent if and only if $\mathbf{v}^{\perp}\left(\mathcal{M}_{1}-\mathcal{M}_{2}\right)=0$.

Example 5.12. The elliptic case $N \leqslant 5$ and the parabolic case $N=6$ are treated in detail in Sections 6 and 7 below, while the range $7 \leqslant N \leqslant 10$ remains open. A few examples are given in Table 3; they were found by the coset enumeration procedure described in Subsection 5.4 and Remark 5.11. All groups listed are $\mathbb{S}$ transitive. The notation is the same as in Table 1: the triples that appear in the Alexander polynomials of genuine trigonal curves are marked with $\mathrm{a}^{*}$, and the last column describes the projection to $\Gamma$ of the corresponding universal subgroup. (This time, the universal subgroups in $\mathbb{B}_{3}$ and in $\mathrm{Bu}_{3}$ have the same projections to $\Gamma$; they differ by their depths.)

Conjecturally, Tables 1 and 3 list all triples $\left(p, N, \varkappa_{\xi}\right), N \geqslant 7$ (including the case $p=0$ ), that appear in the extended Alexander polynomials of subgroups of $\mathrm{Bu}_{3}$, not necessarily $\mathbb{S}$-transitive, of genus zero. The proof, in its current state, requires a great deal of computation and a number of technical details still need to be double checked. It will appear elsewhere. 


\section{Proof of Theorem 1.2}

In this section, we list all roots $\xi$ of the extended Alexander polynomials with $N:=\operatorname{ord}(-\xi) \leqslant 5$. Only finitely many universal subgroups appear, and they are all congruence subgroups of genus zero. Then we eliminate the remaining cases $6 \leqslant N \leqslant 10$ for $p=0$ and prove Theorem 1.2 .

\subsection{Reduction to congruence subgroups}

For an integer $N \geqslant 2$, denote by $\mathbb{B}_{3}(N) \subset \mathbb{B}_{3}$ the subgroup normally generated by $\sigma_{1}^{N}$. For $N \leqslant 5$ these subgroups are of finite index: one has $\operatorname{pr}_{\Gamma} \mathbb{B}_{3}(N)=\Gamma(N)$ and $\operatorname{dp} \mathbb{B}_{3}(N)=6,12,24$, and 60 for $N=2,3,4$, and 5 , respectively.

Lemma 6.1. Fix an integer $N \geqslant 2$ and let $\mathrm{A}^{\prime}=\mathrm{A} / \tilde{\varphi}_{N}(-t)$. Then the induced $\mathbb{B}_{3}$-action on $\mathrm{A}^{\prime} \rtimes \mathbb{Z}$ factors through $\mathbb{B}_{3} / \mathbb{B}_{3}(N)$.

Lemma 6.2. In the notation of Lemma 6.1, let $G_{\mathcal{V}}$ be the universal subgroup corresponding to a submodule $\mathcal{V} \subset \mathrm{A}^{\prime}$. Then all cusp widths of $G_{\mathcal{V}}$ divide $N$. If $N \leqslant 5$, then $\bar{G}_{\mathcal{V}} \subset \Gamma$ is a congruence subgroup of level $l \mid N$.

Proof of Lemmas 6.1 and 6.2. Any element conjugate to $\sigma_{1}^{N}$ acts trivially on $\mathrm{A}(\xi)$, see (2.1), and on the product $\mathrm{A}^{\prime} \rtimes \mathbb{Z}$ (as $\sigma_{1}$ preserves $\left.\alpha_{3}\right)$. If $N \leqslant 5$, then $\Gamma(N)$ is normally generated by $\bar{\sigma}_{1}^{N}$.

Lemma 6.3. In the notation of Lemma 6.1, if $N \leqslant 5$, then the action of the quotient $\mathbb{B}_{3} / \mathbb{B}_{3}(N)$ on $\mathrm{A}^{\prime} \rtimes \mathbb{Z}$ is faithful.

Proof. For any $s \in \mathbb{Z}$ one has

$$
\left[\left(\sigma_{2} \sigma_{1}\right)^{3 s}\left(\alpha_{1}\right) \cdot \alpha_{1}^{-1}\right]=\tilde{\varphi}_{s}\left(t^{3}\right)\left[(t-1) \mathbf{e}_{1}+\left(t^{2}-1\right) \mathbf{e}_{2}\right]
$$

hence the depth of the kernel of the action equals that of $\mathbb{B}_{3}(N)$, see above.

Specializing at $t=-1$, one obtains a faithful action of $S L\left(2, \mathbb{Z}_{N}\right)=\tilde{\Gamma} / \tilde{\Gamma}(N)$ on $\mathbb{Z}_{N} \oplus \mathbb{Z}_{N}$; hence, the images of the kernel and of $\mathbb{B}_{3}(N)$ in $\Gamma$ also coincide.

The action of $\mathbb{B}_{3} / \mathbb{B}_{3}(6)$ is also faithful, see Lemma 7.5 , but I do not know whether this statement extends to $N \geqslant 7$.

Using Lemmas 6.1 and 6.2 and the tables of congruence subgroups found in [5], one can easily enumerate all conjugacy classes of submodules $\overline{\mathcal{V}}_{G} \subset \mathrm{A} / \tilde{\varphi}_{N}(-t)$ for $N \leqslant 5$. In Subsections 6.2-6.4 below, we state a few consequences in terms of the specializations $\overline{\mathcal{V}}_{G}(\xi) \subset \mathrm{A}(\xi)$.

\subsection{The cases $N=3$ and 5}

In this subsection, we do not assume a priori that $G$ is of genus zero or $\mathbb{S}$-transitive. For each universal subgroup $G_{\mathcal{V}}$, we indicate only its image $\bar{G}_{\mathcal{V}}$; in each case, the type specification is recovered uniquely (sometimes up to automorphism) using Proposition 2.9 (always $\operatorname{dp} G_{\mathcal{V}}=2 \mathrm{e}_{p}(N)$ ) and Lemmas 4.3, 4.4, and 4.6. 


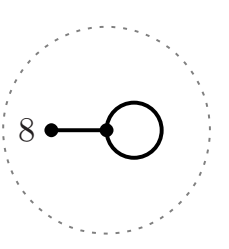

(a) $3 B^{0}\left(\Phi_{6}\right)$

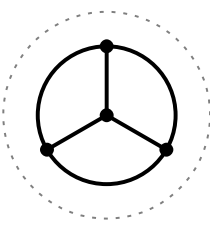

(b) $3 D^{0}\left(\Phi_{6}^{2}\right)$

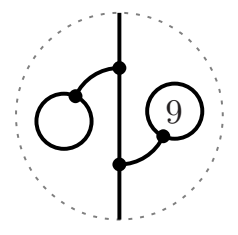

(c) $5 D^{0}\left(\Phi_{10}\right)$

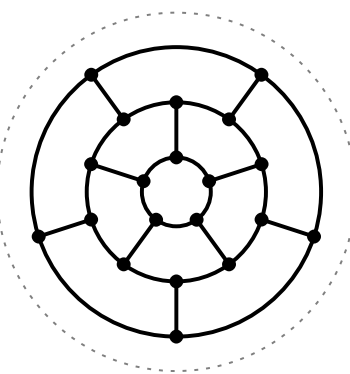

(d) $5 H^{0}\left(\Phi_{10}^{2}\right)$

FIgURE 1. Skeletons of the universal groups for $p=0$.

Theorem 6.5. Assume that the extended Alexander polynomial $\bar{\Delta}_{G, p}$ has a root $\xi \in \mathbb{K} \supset \mathbb{k}_{p}, p \neq 3$, with ord $(-\xi)=3$. Then one has one of the following three mutually exclusive cases:

1. $\overline{\mathcal{V}}_{G}(\xi)=0$; then $\bar{G} \prec \Gamma(3)=3 D^{0}$, Figure $1(\mathrm{~b})$, and unless $p=2$, one has $\overline{\mathcal{V}}_{G}=0 \bmod \Phi_{6}\left(\right.$ hence $\overline{\mathcal{V}}_{G}(\xi)=0$ for any $\left.q\right)$;

2. $\overline{\mathcal{V}}_{G}(\xi) \sim \mathbb{K} \mathbf{e}_{2}$; then $\bar{G} \prec \Gamma_{1}(3)=3 B^{0}$, Figure $1(\mathrm{a})$, and unless $p=2$, one has $\overline{\mathcal{V}}_{G} \sim \Lambda \mathbf{e}_{2} \bmod \Phi_{6}$ (hence $\overline{\mathcal{V}}_{G}(\xi) \sim \mathbb{K}_{q} \mathbf{e}_{2}$ for any $\left.q\right)$;

3. $p=2$ and $\overline{\mathcal{V}}_{G}(\xi)=\mathbb{K}\left(-\right.$ te $\left.\mathbf{e}_{1}+\mathbf{e}_{2}\right)$; then $G \prec(\Gamma)^{-}$, see Proposition 4.7 .

If $G \subset \mathbb{B}_{3}$, then Case (3) does not occur and in all other cases $2 \mathcal{V}_{G} \subset \overline{\mathcal{V}}_{G} \bmod \Phi_{6}$ and $\mathcal{V}_{G}(\xi)=\overline{\mathcal{V}}_{G}(\xi)$ unless $p=2$.

The reason for the exception in Cases (1) and (2) is the fact that, for $p \neq 2$, the type specification is defined modulo $2 \mathrm{e}_{p}(3)=12$, whereas for $p=2$ it is only defined modulo $2 \mathrm{e}_{2}(3)=6$. Hence, the corresponding universal groups are larger (index 2 extensions) for $p=2$. The same remark applies to Theorem 6.7 below.

Proof. Assuming $G$ universal and using Lemma 6.2 , one concludes that $\bar{G}$ is a congruence subgroup of level 1 or 3 , and the submodules $\overline{\mathcal{V}}_{G}(\xi)$ can be computed using the list found in [5]. For $\bar{G}=\Gamma(3)$ and $\Gamma_{1}(3)$, one has $\overline{\mathcal{V}}_{G}=\Phi_{6} \mathrm{~A}$ and $\Lambda \mathbf{e}_{2}+\Phi_{6} \mathrm{~A}$, respectively, $c f$. [6]. The three other subgroups $3 C^{0} \subset 3 A^{0} \subset \Gamma$ have 2-torsion. Hence $\overline{\mathcal{V}}_{G}(\xi)=\mathrm{A}(\xi)$ unless $p=2$, see Lemma 4.6(1). If $p=2$, the universal subgroup is given by Proposition 4.7 .

Cases (2) and (3) are mutually exclusive since the largest subgroup of $\Gamma_{1}(3)$ on which the type specification shown in Figure 1 (a) matches $-\operatorname{dg} \bmod 6$ is $\Gamma(3)$, see Remark 4.9. For the last statement, it suffices to notice that the ideal generated by $\Phi_{6}$ and $t^{2}+t+1$ contains $2 \Lambda$, hence $2 \mathcal{V}_{G} \subset \overline{\mathcal{V}}_{G} \bmod \Phi_{6}$, see Lemma 2.17.

Remark 6.6. If $p=2$ in Theorem 6.5, the module $\mathcal{V}_{G}(\xi)$ depends on the type specification, i.e., on the lift of $G$, which can be regarded as a subgroup of $\operatorname{PSL}\left(2, \mathbb{F}_{3}\right)=$ $\Gamma / \Gamma(3)$, to $S L\left(2, \mathbb{F}_{3}\right)=\mathbb{B}_{3} / \mathbb{B}_{3}(3)$. In other words, $\overline{\mathcal{V}}_{G}(\xi)$ defines the type specification modulo 6 , whereas $\mathcal{V}_{G}(\xi)$ depends on its values modulo 12 . 


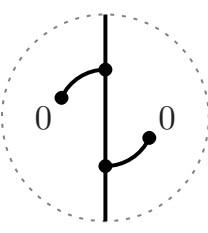

(a) $4 D^{0}$

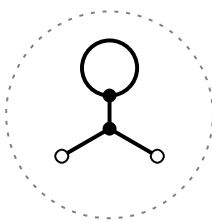

(b) $5 B^{0}$

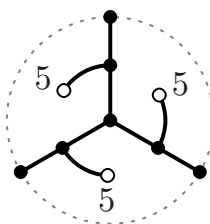

(c) $5 E^{0}$

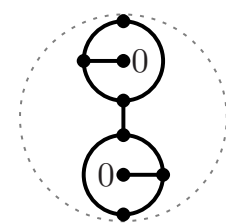

(d) $5 F^{0}$

Figure 2. Some subgroups of level 3, 4, and 5

Theorem 6.7. Assume that the extended Alexander polynomial $\bar{\Delta}_{G, p}$ has a root $\xi \in \mathbb{K} \supset \mathbb{k}_{p}, p \neq 5$, with $\operatorname{ord}(-\xi)=5$. Then one has one of the following four mutually exclusive cases:

1. $\overline{\mathcal{V}}_{G}(\xi)=0$; then $\bar{G} \prec \Gamma(5)=5 H^{0}$, Figure $1(\mathrm{~d})$, and unless $p=2$, one has $\overline{\mathcal{V}}_{G}=0 \bmod \Phi_{10}\left(\right.$ hence $\overline{\mathcal{V}}_{G}(\xi)=0$ for any $\left.q\right)$;

2. $\overline{\mathcal{V}}_{G}(\xi) \sim \mathbb{K} \mathbf{e}_{2}$; then $\bar{G} \prec \Gamma_{1}(5)=5 D^{0}$, Figure 1 (c), and unless $p=2$, one has $\overline{\mathcal{V}}_{G} \sim \Lambda \mathbf{e}_{2} \bmod \Phi_{10}\left(\right.$ hence $\overline{\mathcal{V}}_{G}(\xi) \sim \mathbb{K}_{q} \mathbf{e}_{2}$ for any $\left.q\right)$;

3. $p=2$ and $\overline{\mathcal{V}}_{G}(\xi) \sim \mathbb{K}\left(t^{2} \mathbf{e}_{1}+\mathbf{e}_{2}\right)$; then $\bar{G} \prec 5 E^{0}$, Figure $2(\mathrm{c})$;

4. $p=3$ and $\overline{\mathcal{V}}_{G}(\xi) \sim \mathbb{K}\left(\mathbf{e}_{1}-\right.$ te $\left._{2}\right)$; then $\bar{G} \prec 5 F^{0}$, Figure $2(\mathrm{~d})$.

If $G \subset \mathbb{B}_{3}$, then $\mathcal{V}_{G}=\overline{\mathcal{V}}_{G} \bmod \Phi_{10}$.

Proof. As above, using Lemma 6.2 one can assume that $\bar{G} \supset \Gamma(5)$ and use the list found in [5]. The two torsion free subgroups $\bar{G}=\Gamma(5)$ and $\Gamma_{1}(5)$ result in $\overline{\mathcal{V}}_{G}=0 \bmod \Phi_{10}$ and $\Lambda \mathbf{e}_{2} \bmod \Phi_{10}$, respectively. All other subgroups have torsion and, due to Lemmas 4.4(1) and 4.6(1), one has $\overline{\mathcal{V}}_{G}(\xi)=\mathrm{A}(\xi)$ whenever $p \neq 3$ and $G$ has 3-torsion or $p \neq 2$ and $G$ has 2-torsion.

Assume that $p=2$. The three level 5 subgroups with only 2-torsion are $5 B^{0}$ and $5 G^{0} \subset 5 E^{0}$. The skeleton of $5 B^{0}$, see Figure $2(\mathrm{~b})$, contradicts Lemma 5.1. For the other two groups, a direct computation shows that $\overline{\mathcal{V}}_{G}(\xi)=\mathbb{K}\left(t^{2} \mathbf{e}_{1}+\mathbf{e}_{2}\right)$.

Assume that $p=3$. The only level 5 subgroup with only 3 -torsion is $5 F^{0}$, see Figure $2(\mathrm{~d})$. Over $\Gamma(5)$, it is generated by $\bar{\sigma}_{2} \bar{\sigma}_{1}$ and, lifting this element to $t^{4}\left(\sigma_{2} \sigma_{1}\right)$, one obtains $\overline{\mathcal{V}}_{G}(\xi)=\mathbb{K}\left(\mathbf{e}_{1}-t \mathbf{e}_{2}\right)$, see Subsection 4.3.

Cases (2), (3), and (4) are mutually exclusive since the only common subconjugate of any pair of corresponding universal subgroups is $\Gamma(5)$.

The last statement follows directly from Corollary 2.18.

\subsection{The cases $N=1,2$, and 4}

Here, we do assume that the $\operatorname{subgroup} G$ is $\mathbb{S}$-transitive. Without this assumption, the number of cases in Theorems $6.8,6.9$, and 6.10 would be much larger. As above, we only indicate the image $\bar{G}_{\mathcal{V}}$ of the universal subgroup $G_{\mathcal{V}}$; the type specification is given by Proposition 2.9 and Lemmas 4.3, 4.4, and 4.6.

Theorem 6.8. For an $\mathbb{S}$-transitive subgroup $G$, assume that $\bar{\Delta}_{G, p}(1)=0$. Then:

1. $p=3, \overline{\mathcal{V}}_{G}(1)=\mathbb{K}\left(-t \mathbf{e}_{1}+\mathbf{e}_{2}\right)$, and $G \prec\left(2 A^{0}\right)^{\text {bu }}$, see Proposition $4.8(1)$.

The conventional Alexander polynomial $\Delta_{G, p}$ cannot vanish at 1 . 
Proof. Using Lemma 6.2 , one can assume that $\bar{G} \supset \Gamma(2)$ and, for $G$ to be $\mathbb{S}$ transitive, $\bar{G}$ must not lie in $\Gamma_{1}(2)$, i.e., it must contain $\Gamma^{2}=2 A^{0}$, see [5]. Thus, the statement about $\overline{\mathcal{V}}_{G}(1)$ follows from Propositions 4.7 and 4.8 , and a simple computation for $\bar{G}=2 A^{0}$ (using the fact that over $\Gamma(2)$ this subgroup is generated by $\left.\bar{\sigma}_{2} \bar{\sigma}_{1}\right)$ shows that $\mathcal{V}_{G}(1)=\mathrm{A}(1)$.

Theorem 6.9. For an $\mathbb{S}$-transitive subgroup $G$, assume that $\bar{\Delta}_{G, p}(-1)=0$. Then one has one of the following five mutually exclusive cases:

1. $p=3$ and $\overline{\mathcal{V}}_{G}(-1)=0$; then $\bar{G} \prec \Gamma(3)=3 D^{0}$, Figure $1(\mathrm{~b})$;

2. $p=3$ and $\overline{\mathcal{V}}_{G}(-1) \sim \mathbb{k} \mathbf{e}_{2}$; then $\bar{G} \prec \Gamma_{1}(3)=3 B^{0}$, Figure 1 (a);

3. $p=5$ and $\overline{\mathcal{V}}_{G}(-1)=0$; then $\bar{G} \prec \Gamma(5)=5 H^{0}$, Figure $1(\mathrm{~d})$;

4. $p=5$ and $\overline{\mathcal{V}}_{G}(-1) \sim \mathbb{k} \mathbf{e}_{2}$; then $\bar{G} \prec \Gamma_{1}(5)=5 D^{0}$, Figure 1 (c);

5. $p=7$ and $\overline{\mathcal{V}}_{G}(-1) \sim \mathbb{k} \mathbf{e}_{2}$; then $\bar{G} \prec \Gamma_{1}(7)=7 E^{0}$.

If $G \subset \mathbb{B}_{3}$, then $\mathcal{V}_{G}=\overline{\mathcal{V}}_{G} \bmod (t+1)$.

Proof. Essentially, the statement is the principal result of [6], where all modules $\mathrm{A}_{G} /(t+1)$ are classified. (Note that Lemma 6.2 does not apply to $N=1$.) The action $\mathrm{Bu}_{3}$ on $\mathrm{A} /(t+1)$ factors through $\tilde{\Gamma}$, and the universal subgroups are of the form $\tilde{\Gamma}_{m}(n)$. There are five such subgroups of genus zero that are $\mathbb{S}$-transitive. The last statement follows from Corollary 2.18.

Theorem 6.10. For an $\mathbb{S}$-transitive subgroup $G \subset \mathrm{Bu}_{3}$, assume that $\bar{\Delta}_{G, p}$ has a root $\xi \in \mathbb{K} \supset \mathbb{k}_{p}, p \neq 2$, with $\operatorname{ord}(-\xi)=4$. Then one has:

1. $p=3, \overline{\mathcal{V}}_{G}(\xi) \sim \mathbb{K}\left(\mathbf{e}_{1}-\mathbf{e}_{2}\right)$, and $\bar{G} \prec 4 D^{0}$, Figure 2 (a).

If $G \subset \mathbb{B}_{3}$, then $\mathcal{V}_{G}=\overline{\mathcal{V}}_{G} \bmod \left(t^{2}+1\right)$.

Proof. Using Lemma 6.2, one can assume that $\bar{G}$ is a congruence subgroup of level 2 or 4 . According to [5], there are three $\mathbb{S}$-transitive (i.e., not subconjugate to $\left.\Gamma_{1}(2)\right)$ subgroups with this property: $\Gamma^{2}=2 A^{0}, 4 A^{0}$, and $4 D^{0}$. All three have 3-torsion; hence $\overline{\mathcal{V}}_{G}(\xi)=\mathrm{A}(\xi)$ unless $p=3$, see Lemma 4.4 (1). The subgroup $2 A^{0}$ was considered in Subsection 4.5. The subgroup $4 A^{0}$ has 2 -torsion as well and is eliminated by Lemma $4.6(1)$. The remaining subgroup $4 D^{0}$, see Figure $2(\mathrm{a})$, is generated over $\Gamma(4)$ by $\bar{\sigma}_{2} \bar{\sigma}_{1}$; lifting it to $t^{3}\left(\sigma_{2} \sigma_{1}\right)$ and using Subsection 4.3 , one obtains $\overline{\mathcal{V}}_{G}(\xi)=\mathbb{K}\left(\mathbf{e}_{1}-t \mathbf{e}_{2}\right)$. The last statement follows from Corollary 2.18.

\subsection{Realizability and dependencies}

We show that most pairs $\left(p, \varkappa_{\xi}\right)$ listed in the previous two sections do appear in the (extended) Alexander polynomials of genuine trigonal curves and that most of them are mutually exclusive.

Theorem 6.11. With the exception of 6.5(3), each case listed in Theorems 6.5, $6.7,6.8,6.9$, and 6.10 can be realized by a subgroup of $\mathbb{B}_{3}$, ergo by a genuine trigonal curve. 
Proof. The type specifications in Theorem 6.5, except Case 6.5(3), are trivial modulo 6. In all the other theorems, one has g.c.d. $(M, 3)=1$ and hence the type specifications can be chosen trivial modulo 6 .

Remark 6.12. The minimal, in the sense of the skeleton, genuine trigonal curve with the Alexander polynomial $\Phi_{10}$ has non-simple singularities. According to the type specification shown in Figure 1 (c), it must be a curve in $\Sigma_{10}$ with the set of singular fibers $\tilde{\mathbf{J}}_{8,0} \oplus 2 \tilde{\mathbf{A}}_{4} \oplus \tilde{\mathbf{A}}_{0}^{*}$.

Theorem 6.13. The fourteen cases listed in Theorems 6.5, 6.7, 6.8, 6.9, and 6.10, are related as follows:

1. if $p \neq 2$, then $6.5(1) \Longrightarrow 6.9(1)$ and $6.5(2) \Longrightarrow 6.9(2)$;

2. if $p \neq 2$, then $6.7(1) \Longrightarrow 6.9(3)$ and $6.7(2) \Longrightarrow 6.9(4)$;

3. $6.10(1) \Longrightarrow 6.8(1)$;

4. Cases $6.5(2) \Longrightarrow 6.9(2)$ and $6.8(1)$ can occur simultaneously;

5. Case 6.5(3) can occur simultaneously with any case except 6.5(1), (2);

6. otherwise, if $G$ is of genus zero, the cases are mutually exclusive.

The implications in (1) turn into equivalences if $G \subset \mathbb{B}_{3}$ and $p \neq 2$.

Proof. For (1) and (2), the universal subgroups coincide, the type specifications in Theorems 6.5 and 6.7 being more restrictive (defined, respectively, modulo 12 or 20) than those in Theorem 6.9 (defined modulo 4 only). If $G \subset \mathbb{B}_{3}$, Proposition 2.9 makes the type specifications in Theorem 6.9 well defined modulo 12 as well (if $p \neq 2$ ) and the implications in (1) turn into equivalences.

The implication in (3) follows from the inclusion $4 D^{0} \subset 2 A^{0}$.

The fact that the cases within each theorem are mutually exclusive is stated in the corresponding theorem. Otherwise, consider two cases and let $G_{1}$ and $G_{2}$ be the corresponding universal subgroups, and $M_{1}$ and $M_{2}$ the values of $M$. For the two cases to occur simultaneously, the projections $\bar{G}_{1}$ and $\bar{G}_{2}$ must have a common subconjugate of genus zero. Then, if in addition g.c.d. $\left(M_{1}, M_{2}\right)=1$, one can also find a common type specification. Common subconjugates can be analyzed using the tables found in [5] (listing, in particular, all sub/supergroups). Unless one of the groups is $\Gamma$ itself (Item (5) of the statement), the only pair is $2 A^{0} \supset 6 C^{0} \subset 3 B^{0}$, which accounts for Item (4).

The following fact was stated in [6] without proof.

Corollary 6.14. For an irreducible genuine trigonal curve $C$, if $\Phi_{M}^{2} \mid \Delta_{C}$, then the fundamental group $\pi_{C}^{\text {afn }}$ admits a dihedral quotient $\mathbb{D}\left(\mathbb{Z}_{M} \oplus \mathbb{Z}_{M}\right)$.

Theorem 6.15. For an $\mathbb{S}$-transitive subgroup $G \subset \mathrm{Bu}_{3}$ of genus zero, assume that $\overline{\mathcal{V}}_{G}(\xi)=0$ for some $\xi \in \mathbb{K} \supset \mathbb{k}_{p}$. Then $\bar{G} \subset \Gamma(N)$ for $N=3$ or 5 , see Cases $6.5(1), 6.7(1)$, and $6.9(1)$ and $(3)$.

Proof. According to Lemma 4.11, $G$ is torsion free and all its cusp widths are divisible by $N$. As a torsion free subgroup of genus zero, $G$ is generated by its para- 
bolic elements, hence $G \subset \Gamma(N)$. Then $N \leqslant 5$, and it remains to observe that the subgroups $\Gamma(4) \subset \Gamma(2)$ are not $\mathbb{S}$-transitive.

\subsection{The case $p=0$ and $\mathrm{e}_{0}(N)=q^{r}$}

Assume that one of the polynomials $\Delta_{G}$ or $\bar{\Delta}_{G}$ of a subgroup $G \subset \mathbb{B}_{3} \cdot \operatorname{Inn} \mathfrak{F}$ has a root $\xi$ of order $M:=\mathrm{e}_{0}(N)=q^{r}$, where $q$ is a prime. In the range $3 \leqslant N \leqslant 10$, see Corollary 5.10, this is the case for $N=4,6,8$, or 10, all even values of $N$.

Lemma 6.16. Let $M=q^{r}$ be a prime power. If $\Phi_{M}$ divides $\Delta_{G}$ or $\bar{\Delta}_{G}$, then $(t-1)$ divides $\Delta_{G, q}$ or $\bar{\Delta}_{G, q}$, respectively.

Proof. We will prove the statement for $\Delta$; the proof for $\bar{\Delta}$ is a literal repetition.

With the stated assumptions, the group $\mathrm{A}_{G} / \Phi_{M}$ is infinite (as it remains nontrivial after tensoring with $\mathbb{Q})$. Hence, the $q$-group $\operatorname{Hom}\left(\mathrm{A}_{G} / \Phi_{M}, \mathbb{F}_{q}\right)$ is nontrivial, and the order $q^{r}$ automorphism $t$ of this group has a nontrivial invariant element $\varphi$. Then the $\left(\Lambda \otimes \mathbb{F}_{q}\right)$-module $\mathrm{A}_{G} \otimes \mathbb{F}_{q}$ factors as $\operatorname{Im} \varphi \cong \mathbb{F}_{q}=\left(\Lambda \otimes \mathbb{F}_{q}\right) /(t-1)$.

Corollary 6.17. Let $M=q^{r}$ be a prime power, and let $G$ be an $\mathbb{S}$-transitive subgroup. Then $\Phi_{M} \nmid \Delta_{G}$, and if $\Phi_{M} \mid \bar{\Delta}_{G}$, one has:

1. $M=3, \overline{\mathcal{V}}_{G}=\Lambda\left(-t \mathbf{e}_{1}+\mathbf{e}_{2}\right) \bmod \Phi_{3}$ and $G \prec\left(2 A^{0}\right)^{-}$, see Proposition 4.8 . $A$ subgroup $G$ with these properties cannot lie in $\mathbb{B}_{3}$.

Proof. The statement follows from Lemma 6.16 and Theorems 6.8 and 7.16.

\subsection{Eliminating $N=7$ and 9 for $p=0$}

Let $\mathbb{k}=\mathbb{Q}$ and let $\xi$ be a primitive root of $(-1)$ of degree 7 or 9 , so that $\varkappa_{\xi}$ is the cyclotomic polynomial $\Phi_{14}$ or $\Phi_{18}$, respectively. Note that, in both cases, $\operatorname{deg} \varkappa_{\xi}=6$, so that $\mathbb{Q}(\xi) \supset \mathbb{Q}$ is a Galois extension of degree six.

Fix a vector $h \in \mathrm{A}(\xi)$ and consider the universal subgroup

$$
G_{h}:=\left\{\beta \in \mathrm{Bu}_{3} \mid \operatorname{Im}[\beta(\xi)-\mathrm{id}] \subset \Lambda(\xi) h\right\} \subset \mathrm{Bu}_{3} .
$$

Lemma 6.18. For any $h \in \mathrm{A}(\xi)$, one has $\left[\mathrm{Bu}_{3}: G_{h}\right]=\left[\Gamma: \bar{G}_{h}\right]=\infty$.

Proof. Consider the element

$$
\beta:=t \sigma_{1}^{-1} \sigma_{2}=\left[\begin{array}{cc}
t-1 & -t \\
t^{2} & -t^{2}
\end{array}\right]
$$

and its specialization $\beta(\xi)$. We assert that, in an appropriate extension $\mathbb{K} \supset \mathbb{Q}(\xi)$ of degree at most two, $\beta(\xi)$ has two distinct eigenvalues which are not roots of unity. Indeed, the characteristic polynomial of $\beta(\xi)$ is $\chi(\lambda)=\lambda^{2}+\left(\xi^{2}-\xi+1\right) \lambda+\xi^{2}$ and its roots belong to an extension of $\mathbb{Q}$ of degree 6 or 12 . Hence, the degree of the minimal polynomial $\varkappa_{\lambda} \in \mathbb{Q}[t]$ of any eigenvalue $\lambda$ divides 12 . There are finitely many cyclotomic polynomials $\Phi_{n}$ with $\operatorname{deg} \Phi_{n} \mid 12$ (one has $n=1,2,3,4,5,6$, $7,8,9,10,12,13,14,18,21,26,28,36$, or 42 ; alternatively, in the computation below one can use the polynomials $\lambda^{n}-1$ with $n=8,10,26,28,36$, or 42). For each such polynomial $\Phi_{n}(\lambda)$, compute the resultant $\mathcal{R}_{n}(\xi)$ of $\Phi_{n}(\lambda)$ and $\chi(\lambda)$ with 
respect to $\lambda$, treating $\xi$ as an independent variable. Each time, it is straightforward that $\mathcal{R}_{n} \bmod \varkappa_{\xi} \neq 0$; hence $\chi(\lambda)$ and $\Phi_{n}(\lambda)$ have no common roots in any extension of $\mathbb{Q}(\xi)$. (This computation was performed using Maple.) It follows that the two roots of $\chi$ are not roots of unity and, in particular, they are distinct (as their product $\xi^{2}$ is a root of unity).

Thus, for any pair of integers $m \neq 0$ and $r$, the two eigenvalues of $\xi^{r} \beta^{m}(\xi)-$ id are distinct and nonzero. Hence, $\operatorname{rk}\left[\xi^{r} \beta^{m}(\xi)-\mathrm{id}\right]=2$ and $t^{r} \beta^{m} \notin G_{h}$. On the other hand, the projection $\bar{\beta} \in \Gamma$ is an element of infinite order.

Corollary 6.19. If $G \subset \mathrm{Bu}_{3}$ and $[\Gamma: \bar{G}]<\infty$ (e.g., if $G$ is a subgroup of genus zero), the polynomial $\bar{\Delta}_{G}$ is not divisible by $\Phi_{14}$ or $\Phi_{18}$.

Remark 6.20. In the proof of Lemma 6.18, we used Maple to show that a certain algebraic number is not a root of unity. Probably there should be a better way to detect rational arguments, and I expect that the statement of the lemma holds for any primitive root $\xi \in \mathbb{C}$ of $(-1)$ of degree $N \geqslant 7$.

\subsection{Proof of Theorem 1.2}

Let $G \subset \mathbb{B}_{3} \cdot \operatorname{Inn} \mathfrak{F}$ be the monodromy group of the curve; it is an $\mathbb{S}$-transitive subgroup of genus zero, see Theorem 3.2. Due to Lemma 4.10 and Corollary 5.10, each irreducible factor of $\Delta_{G}$ is of the form $\Phi_{M}$ with $N:=\mathrm{e}_{0}(M) \leqslant 10$. Most values of $N$ are eliminated above, see Theorem 6.8 for $N=2$, Theorem 6.9 for $N=1$, Corollary 6.17 for $N=4,6,8$, and 10, and Corollary 6.19 for $N=7,9$. The multiplicity of each of the remaining factors $\Phi_{6}$ and $\Phi_{10}$ cannot exceed two, see Lemma 4.10, and two distinct factors cannot appear simultaneously according to Theorem 6.13 . The realizability is given by Theorem 6.11 , see Remark 3.5 .

\section{The case $N=6$}

In this section, we treat the parabolic case $N:=\operatorname{ord}(-\xi)=6$. Since $\xi^{2}+\xi+1=0$ in this case, Corollary 2.18 does not apply and we consider the extended modules $\overline{\mathcal{V}}_{G} \subset \mathrm{A}$ and $\overline{\mathrm{A}}_{G}$ only.

\subsection{The action on $\mathbf{A}^{\prime}$}

Let $\Lambda^{\prime}=\Lambda / \Phi_{3}$ and $\mathrm{A}^{\prime}=\mathrm{A} / \Phi_{3}$; for an integer $m>1$, let also $\Lambda_{m}^{\prime}=\Lambda^{\prime} \otimes \mathbb{Z}_{m}$ and $\mathrm{A}_{m}^{\prime}=\mathrm{A}^{\prime} \otimes \mathbb{Z}_{m}$. Consider the vector $\mathbf{v}=-t \mathbf{e}_{1}+\mathbf{e}_{2} \in \mathrm{A}^{\prime}$. It is immediate that $\sigma_{1}(\mathbf{v})=\sigma_{2}(\mathbf{v})=\mathbf{v}$, and in the basis $\left\{\mathbf{v}, \mathbf{e}_{2}\right\}$ the induced $\mathbb{B}_{3}$-action is given by the matrices

$$
\sigma_{1}=\left[\begin{array}{cc}
1 & -t^{2} \\
0 & -t
\end{array}\right], \quad \sigma_{2}=\left[\begin{array}{cc}
1 & 0 \\
0 & -t
\end{array}\right]
$$

hence

$$
\sigma_{1} \sigma_{2}^{-1}=\left[\begin{array}{cc}
1 & t \\
0 & 1
\end{array}\right], \quad \sigma_{2}^{-1} \sigma_{1}=\left[\begin{array}{cc}
1 & t+1 \\
0 & 1
\end{array}\right] .
$$

It follows that the image of the action on $\mathrm{A}^{\prime}$ is the full group of upper triangular matrices with $\left[1,(-t)^{s}\right]$ in the diagonal. 
Let $\Gamma^{\prime}=[\Gamma, \Gamma]=6 A^{1}$. Recall that it is the free subgroup generated by $\bar{\sigma}_{1} \bar{\sigma}_{2}^{-1}$ and $\bar{\sigma}_{2}^{-1} \bar{\sigma}_{1}$. Let $\Gamma^{\prime \prime}=\left[\Gamma^{\prime}, \Gamma^{\prime}\right]$ be the second commutant, and let $\Gamma_{m}^{\prime \prime} \subset \Gamma^{\prime}$ be the preimage of $m \mathbb{Z} \oplus m \mathbb{Z}$ under the abelianization homomorphism $\Gamma^{\prime} \rightarrow \mathbb{Z} \oplus \mathbb{Z}$.

The next two lemmas follow immediately from (7.1) and (7.2).

Lemma 7.3. The kernels of the $\mathbb{B}_{3}$-actions on $\mathrm{A}^{\prime}$ and $\mathrm{A}_{m}^{\prime}$ are the subgroups $\left(\Gamma^{\prime \prime}\right)^{+}$ and $\left(\Gamma_{m}^{\prime \prime}\right)^{+}$, respectively. The image of $\left(\Gamma^{\prime}\right)^{+} /\left(\Gamma^{\prime \prime}\right)^{+}$in $S L\left(\mathrm{~A}^{\prime}\right)$ consists of all unipotent upper triangular matrices.

Lemma 7.4. Any two vectors of the form $\mathbf{e}_{2}+f_{i} \mathbf{v} \in \mathrm{A}^{\prime}, f_{i} \in \Lambda^{\prime}, i=1,2$, are conjugate to each other.

Lemma 7.5. The action of $\mathbb{B}_{3} / \mathbb{B}_{3}(6)$ on $\mathrm{A}^{\prime} \rtimes \mathbb{Z}$, see Lemma 6.1 , is faithful.

Proof. The images in $\Gamma$ of the elements $\beta_{1}:=\sigma_{1} \sigma_{2}^{-1}$ and $\beta_{2}:=\sigma_{2}^{-1} \sigma_{1}$ generate $\Gamma^{\prime}$, and the image of the commutator $\left[\beta_{1}, \beta_{2}\right]:=\beta_{1} \beta_{2} \beta_{1}^{-1} \beta_{2}^{-1}$ normally generates $\Gamma^{\prime \prime}$. One can easily check the identity $\left[\beta_{1}, \beta_{2}\right]\left(\sigma_{2} \sigma_{1}\right)^{-3}=\sigma_{1} \sigma_{2}^{-6} \sigma_{1}^{-1}$; hence $\mathbb{B}_{3}(6)$ is the lift $\left(\Gamma^{\prime \prime}\right)^{0}$ of $\Gamma^{\prime \prime}$ with the slope $0: \Gamma^{\prime \prime} \rightarrow \mathbb{Z}$. On the other hand, due to $(6.4)$, the kernel of the action is a subgroup of depth 0 .

Lemma 7.6. If $m \neq 0 \bmod 3$, the subgroup $\Gamma_{m}^{\prime \prime}$ is $\mathbb{S}$-transitive.

Proof. One has $\left(\bar{\sigma}_{1} \bar{\sigma}_{2}^{-1}\right)^{m} \in \Gamma_{m}^{\prime \prime}$.

Lemma 7.7. Any subgroup $G \subset \Gamma$ containing $\Gamma_{m}^{\prime \prime}$ is of genus at most one. If $m$ is prime to 6 , the following statements are equivalent:

1. $G$ is of genus zero;

2. $[\Gamma: G] \neq 0 \bmod 6$;

3. $G \not \subset \Gamma^{\prime}$;

4. G has torsion.

Proof. The group $\Gamma^{\prime}$ is torsion free and all its cusp widths are equal to 6 . According to Lemma 6.2 , all cusp widths of $\Gamma_{m}^{\prime \prime}$ divide 6 . Hence, the covering $\mathrm{Sk}_{\Gamma_{m}^{\prime \prime}} \rightarrow \mathrm{Sk}_{\Gamma^{\prime}}$ is unramified, see Subsection 2.4.4, and $\Gamma_{m}^{\prime \prime}$ has genus one (as $\Gamma^{\prime}$ has genus one and any unramified covering of a torus is a torus).

One has $\left[\Gamma: \Gamma^{\prime}\right]=6$ and $\left[\Gamma^{\prime}: G^{\prime}\right] \mid m^{2}$ for any $G^{\prime} \subset \Gamma^{\prime}$; hence, statements (2) and (3) are equivalent. Obviously, (2) implies (4), and (4) implies (3), as $\Gamma^{\prime}$ is torsion free. Since $\Gamma^{\prime}$ has genus one, (1) implies (3). Finally, if $G$ has torsion, the covering $\mathrm{Sk}_{G \cap \Gamma^{\prime}} \rightarrow \mathrm{Sk}_{G}$ is ramified, see Subsection 2.4.4, and $G$ has genus zero (as any ramified covering by a torus has sphere as the base). Thus, (4) implies (1).

\subsection{Subgroups of $\mathbb{B}_{3}$}

In this and the following subsections, we treat the case of genuine trigonal curves, i.e., we assume that $G \subset \mathbb{B}_{3}$.

Lemma 7.8. Assume that $m$ is prime to 6 . For a subgroup $G \subset \mathbb{B}_{3}$, denote by $\overline{\mathcal{V}}_{G}^{\prime}$ the projection of $\overline{\mathcal{V}}_{G}$ to $\mathrm{A}_{m}^{\prime}$. Then either

1. $\overline{\mathcal{V}}_{G}^{\prime}=0 \bmod \Lambda_{m}^{\prime} \mathbf{v}$, and then $\bar{G} \subset \Gamma^{\prime}$, or

2. $\overline{\mathcal{V}}_{G}^{\prime}=\mathrm{A}_{m}^{\prime} \bmod \Lambda_{m}^{\prime} \mathbf{v}$, and then $\bar{G} \not \subset \Gamma^{\prime}$. 
Conversely, any submodule $\mathcal{V} \subset \mathrm{A}_{m}^{\prime}$ satisfying (1) or (2) above is of the form $\overline{\mathcal{V}}_{G}^{\prime}$ for some subgroup $G \subset \mathbb{B}_{3}$.

Proof. All statements follow immediately from the description of the action via upper triangular matrices, see (7.1) and (7.2), and the fact that all polynomials $(-t)^{s}-1, s \neq 0 \bmod 6$, are invertible in $\Lambda_{m}^{\prime}$.

Theorem 7.9. Let $G \subset \mathbb{B}_{3}$ be a subgroup of genus zero. Then the module $\mathrm{A}_{G}^{\prime}:=$ $\mathrm{A}_{G} / \Phi_{3}$ is finite and, modulo 2- and 3-torsion, one has

1. $\mathrm{A}_{G}^{\prime}=\mathrm{A}_{m}^{\prime} /\left(\Lambda_{m}^{\prime} \mathbf{e}_{2}+I \mathbf{v}\right)$ for some integer $m$ prime to 6 and ideal $I \subset \Lambda_{m}^{\prime}$. Conversely, any module $\mathrm{A}_{m}^{\prime} /\left(\Lambda_{m}^{\prime} \mathbf{e}_{2}+I \mathbf{v}\right)$ as above is of the form $\mathrm{A}_{G}^{\prime}$ for some $\mathbb{S}$-transitive genus zero subgroup $G \subset \mathbb{B}_{3}$.

Proof. One has $\left(\sigma_{1} \sigma_{2}^{-1}\right)^{s} \mathbf{e}_{2}-\mathbf{e}_{2}=s t \mathbf{v}$ for any $s \in \mathbb{Z}$. Hence, for $\mathrm{A}_{G}^{\prime}$ to be infinite, the submodule $\overline{\mathcal{V}}_{G} / \Phi_{3}$ must lie in $\Lambda^{\prime} \mathbf{v}$. Then Lemma 7.8 implies that $\bar{G} \subset \Gamma^{\prime}$ is a subgroup of genus at least one.

Assume that $\mathrm{A}_{G}^{\prime}$ is finite. Then, modulo 2- and 3-torsion, $\mathrm{A}_{G}^{\prime}=\mathrm{A}_{m}^{\prime} / \overline{\mathcal{V}}_{G}^{\prime}$ for some sufficiently large $m$ prime to 6 . Since $\bar{G} \not \subset \Gamma^{\prime}$, one has $\overline{\mathcal{V}}_{G}^{\prime}=\mathrm{A}_{m}^{\prime} \bmod \Lambda_{m}^{\prime} \mathbf{v}$, see Lemma 7.8, i.e., $\overline{\mathcal{V}}_{G}^{\prime}$ contains a vector of the form $\mathbf{e}_{2}+f \mathbf{v}, f \in \Lambda_{m}^{\prime}$. In view of Lemma 7.4, any such vector is conjugate to $\mathbf{e}_{2}$, i.e., up to conjugation $\overline{\mathcal{V}}_{G}^{\prime}$ is as stated in the theorem.

Conversely, any submodule $\mathcal{V}=\Lambda_{m}^{\prime} \mathbf{e}_{2}+I \mathbf{v} \subset \mathrm{A}_{m}^{\prime}$ as in the statement is of the form $\overline{\mathcal{V}}_{G}^{\prime}$ for some subgroup $G \subset \mathbb{B}_{3}$ with $\Gamma_{m}^{\prime \prime} \subset \bar{G} \not \subset \Gamma^{\prime}$, see Lemma 7.8; this subgroup is $\mathbb{S}$-transitive, Lemma 7.6, and has genus zero, Lemma 7.7.

\subsection{A characterization of universal subgroups}

Let $\mathrm{Sk}$ be the skeleton of a genus zero subgroup $G \subset \Gamma$. Assume that it has only 1, 2, 3, and 6-gonal regions. Then Euler's formula yields

$$
3 n_{\circ}+4 n_{\bullet}+5 n_{1}+4 n_{2}+3 n_{3}=12,
$$

where $n_{\circ}, n_{\bullet}$, and $n_{i}, i=1,2,3,6$ are, respectively, the numbers of monovalent oand $\bullet$-vertices and $i$-gonal regions of Sk. One of the solutions to this equation is $n_{\circ}=n_{\bullet}=n_{1}=1, n_{2}=n_{3}=0$, and in this case one has $[\Gamma: G]=6 n_{6}+1$.

Definition 7.11. A proper finite index subgroup $G$ of $\mathbb{B}_{3}$ (or of $\Gamma$ ) is called 6significant if $\operatorname{dp} G=6$ and the skeleton $\mathrm{Sk}$ of $G$ has exactly two monovalent vertices, one o- and one $\bullet$-, and one monogonal region, while all other regions of Sk are hexagons.

Note that any 6-significant subgroup $G \subset \mathbb{B}_{3}$ is automatically $\mathbb{S}$-transitive and of genus zero. Note also that, $\operatorname{since} \operatorname{dp} G=6$, it is not important whether we speak about subgroups of $\mathbb{B}_{3}$ or $\Gamma$ : one always has $G=(\bar{G})^{+}$. Examples of 6 -significant subgroups are shown in Figure 3.

Lemma 7.12. Any 6-significant subgroup $G \subset \Gamma$ contains $\Gamma_{m}^{\prime \prime}$ for some integer $m$ prime to 6 . One can take $m=[\Gamma: G]$.

Proof. Since $[\Gamma: G]=6 n_{6}+1=1 \bmod 6$, see above, the subgroup $G^{\prime}:=G \cap \Gamma^{\prime}$ is of index 6 in $G$, torsion free, and with all cusp widths equal to $0 \bmod 6$. On the 


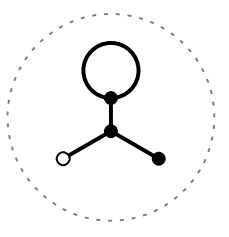

(a) $p=7$

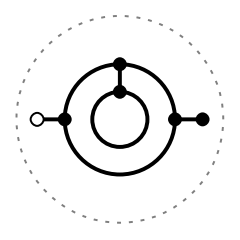

(b) $p=13$

Figure 3. Examples of $G_{\xi}, N=6$.

other hand, there is a unique subgroup of $G$ with these properties: it corresponds to the 6 -fold cyclic covering $\mathrm{Sk}^{\prime} \rightarrow \mathrm{Sk}_{G}$ appropriately ramified over the monovalent vertices and the monogonal region. From the latter description, it follows that all cusp widths of $G^{\prime}$ are equal to 6 . Hence, $\mathrm{Sk}_{G^{\prime}} \rightarrow \mathrm{Sk}_{\Gamma^{\prime}}$ is an unramified covering of degree $m=\left[\Gamma^{\prime}: G^{\prime}\right]=[\Gamma: G]$ and, the fundamental group of the torus being $\mathbb{Z} \times \mathbb{Z}$, it splits the $\left(\mathbb{Z}_{m} \times \mathbb{Z}_{m}\right)$-covering corresponding to the inclusion $\Gamma_{m}^{\prime \prime} \hookrightarrow \Gamma^{\prime}$.

Theorem 7.13. A subgroup $G \subset \mathbb{B}_{3}$ is the universal subgroup corresponding to a proper submodule $\mathcal{V}=\Lambda_{m}^{\prime} \mathbf{e}_{2}+I \mathbf{v} \subset \mathrm{A}_{m}^{\prime}$ for some sufficiently large $m$ prime to 6 , see Theorem 7.9, if and only if it is 6-significant. One can take $m=[\Gamma: \bar{G}]$.

Proof. According to Lemma 7.4, any submodule as in the statement contains vectors conjugate to $-\mathbf{e}_{1}+\mathbf{e}_{2}$ and $t \mathbf{e}_{1}+\mathbf{e}_{2}$. Hence, the universal subgroup has both 2 and 3-torsion and, in view of Lemma 6.2 and (7.10), it is 6-significant.

For the converse, consider a 6-significant subgroup $G$ and let $m=[\Gamma: G]$. Due to Lemmas 7.12 and $7.3, G \supset \Gamma_{m}^{\prime \prime}$ and $G / \Gamma_{m}^{\prime \prime}$ acts faithfully on $\mathrm{A}_{m}^{\prime}$. Up to conjugation, one can assume that $\sigma_{2} \in G$; then $G / \Gamma_{m}^{\prime \prime}$ has the form

$$
G=\left\{\left[\begin{array}{cc}
1 & a \\
0 & (-t)^{s}
\end{array}\right] \mid s \in \mathbb{Z}_{6}, a \in I\right\}
$$

for some ideal $I \subset \Lambda_{m}^{\prime}$, and it is clear that $G$ is the universal subgroup corresponding to the submodule $\mathcal{V}=\Lambda_{m}^{\prime} \mathbf{e}_{2}+I \mathbf{v} \subset \mathrm{A}_{m}^{\prime}$.

Example 7.14. According to Theorems 7.9 and 7.13, there are infinitely many conjugacy classes of 6 -significant subgroups: they can be classified by the proper ideals $I \subset \Lambda^{\prime}$ with the property that $m \Lambda^{\prime} \subset I$ for some $m$ prime to 6 . Using the list in [5], one can see that none of them is a congruence subgroup.

Two examples of 6-significant subgroups are shown in Figure 3. (For the notation, see Theorem 7.16 below.) In each case, the skeleton shown in the figure and its mirror image correspond to the two distinct cubic roots of unity in $\mathbb{F}_{p}$.

\subsection{Subgroups with nontrivial type specification}

In conclusion, we show that the only other source of nontrivial modules $\mathrm{A}_{G}^{\prime}$ is the subgroup $\left(2 A^{0}\right)^{-}$, see Proposition $4.8(2)$.

Theorem 7.15. For a subgroup $G \not \subset \mathbb{B}_{3}$, let $\mathrm{A}_{G}^{\prime}=\mathrm{A}_{G} / \Phi_{3}$ and assume that $6 \mathrm{~A}_{G}^{\prime} \neq 0$. Then $\overline{\mathcal{V}}_{G} \subset \Lambda\left(-t \mathbf{e}_{1}+\mathbf{e}_{2}\right) \bmod \Phi_{3}$ and $G \subset\left(2 A^{0}\right)^{-}$.

If $G$ is $\mathbb{S}$-transitive and has genus zero, then $\overline{\mathcal{V}}_{G}=\Lambda\left(-t \mathbf{e}_{1}+\mathbf{e}_{2}\right) \bmod \Phi_{3}$. 
Proof. Denote by $\overline{\mathcal{V}}_{G}^{\prime}$ the image of $\overline{\mathcal{V}}_{G}$ in $\mathrm{A}^{\prime}$. For any integer $s \neq 0 \bmod 6$, one has $\Lambda^{\prime}\left(t^{s}-1\right) \supset 6 \Lambda^{\prime}$, and it follows from (7.1) that, whenever the type specification is not trivial modulo 6 , there is an inclusion $6 \Lambda^{\prime} \mathbf{v} \subset \overline{\mathcal{V}}_{G}^{\prime}$. The induced $\mathbb{B}_{3}$-action on $\mathrm{A}^{\prime} / \mathbf{v}$ is $\beta: h \mapsto(-t)^{\operatorname{dg} \beta} h$; hence, as above, $\overline{\mathcal{V}}_{G}^{\prime}$ is not a submodule of $6 \mathrm{~A}^{\prime}$ if and only if $\bar{G} \subset 2 A^{0}=\operatorname{Ker}(\operatorname{dg} \bmod 2)$ and the type specification is $-\operatorname{dg} \bmod 6$, i.e., $G \subset\left(2 A^{0}\right)^{-}$.

For $G=\left(2 A^{0}\right)^{-}$, one does have $\overline{\mathcal{V}}_{G}^{\prime}=\Lambda^{\prime} \mathbf{v}$, and for any subgroup $G^{\prime} \subset G$ not contained in $\mathbb{B}_{3}$, still $\overline{\mathcal{V}}_{G^{\prime}} \supset 6 \Lambda^{\prime} \mathbf{v}$. Tensoring the module $\mathrm{A}_{G^{\prime}}^{\prime}$ with $\mathbb{F}_{2}$ or $\mathbb{F}_{3}$ and using Theorems 6.5 and 6.8 , one concludes that, if $G^{\prime}$ is $\mathbb{S}$-transitive and of genus zero, the quotient $\Lambda^{\prime} \mathbf{v} / \overline{\mathcal{V}}_{G^{\prime}}$ cannot have 2 or 3 -torsion. (Note in addition that the intersection $\left(2 A^{0}\right)^{-} \cap \mathbb{B}_{3}=\left(\Gamma^{\prime}\right)^{+}$has genus one, see Remark 4.9.)

To summarize the results obtained in this section, we restate a few consequences of Theorems 7.9 and 7.15 in terms of the specializations $\overline{\mathcal{V}}_{G}(\xi)$.

Theorem 7.16. For a genus zero $\mathbb{S}$-transitive subgroup $G \subset \mathrm{Bu}_{3}$, assume that the extended Alexander polynomial $\bar{\Delta}_{G, p}$ has a root $\xi \in \mathbb{K} \supset \mathbb{k}_{p}, p \neq 2,3$, with $\operatorname{ord}(-\xi)=6$. Then one has one of the following two cases:

1. $\overline{\mathcal{V}}_{G}=\Lambda\left(-t \mathbf{e}_{1}+\mathbf{e}_{2}\right) \bmod \Phi_{3}$ and $G \prec\left(2 A^{0}\right)^{-}$;

2. $p \geqslant 5, \overline{\mathcal{V}}_{G}(\xi) \sim \mathbb{K}_{2}$, and $G \prec\left(G_{\xi}\right)^{+} \subset \mathbb{B}_{3}$, where $G_{\xi} \subset \Gamma$ is a certain subgroup of index $p^{\operatorname{deg} \varkappa \xi}$.

Cases (1) and (2) are mutually exclusive. In Case (2), any finite number of distinct pairs $\left(p, \varkappa_{\xi}\right)$ can appear in the Alexander module of a particular group.

Proof. Cases (1) and (2) are given by Theorems 7.15 and 7.9, respectively; they are mutually exclusive due to Remark 4.9. In Case (2), any finite number of distinct primes $p_{i} \geqslant 5$ can be 'mixed' in the module $\mathrm{A}_{G}=\mathrm{A}_{m}^{\prime} / \Lambda_{m}^{\prime} \mathbf{e}_{2}$, where $m=\prod_{i} p_{i}^{2}$, see Theorem 7.9.

\section{References}

[1] Artin, E.: Theory of braids. Ann. of Math. (2) 48 (1947), 101-126.

[2] Birch, B.: Noncongruence subgroups, covers and drawings. In The Grothendieck theory of dessins d'enfants (Luminy, 1993), 25-46. London Math. Soc. Lecture Note Ser. 200, Cambridge Univ. Press, Cambridge, 1994.

[3] Bogomolov, F. And Tschinkel, Y.: Monodromy of elliptic surfaces. In Galois groups and fundamental groups, 167-181. Math. Sci. Res. Inst. Publ. 41, Cambridge Univ. Press, Cambridge, 2003.

[4] Burau, W.: Über Zopfgruppen und gleichsinnig verdrillte Verkettungen. Abh. Math. Sem. Univ. Hamburg 11 (1935), 179-186.

[5] Cummins, C. J. And Pauli, S.: Congruence subgroups of $\operatorname{PSL}(2, \mathbb{Z})$ of genus less than or equal to 24. Experiment. Math. 12 (2003), no. 2, 243-255.

[6] Degtyarev, A.: Dihedral coverings of trigonal curves. Indiana Univ. Math. J. 61 (2012), no. 3, 901-938. 
[7] Degtyarev, A.: Alexander polynomial of a curve of degree six. J. Knot Theory Ramifications 3 (1994), no. 4, 439-454.

[8] Degtyarev, A.: A divisibility theorem for the Alexander polynomial of a plane algebraic curve. Zap. Nauchn. Sem. S.-Peterburg. Otdel. Mat. Inst. Steklov. (POMI) 280 (2001), Geom. i Topol. 7, 146-156, 300. English translation: J. Math. Sci. (N. Y.) 119 (2004), no. 2, 205-210.

[9] Degtyarev, A.: Oka's conjecture on irreducible plane sextics. J. Lond. Math. Soc. (2) 78 (2008), no. 2, 329-351.

[10] Degtyarev, A.: The fundamental group of a generalized trigonal curve. Osaka J. Math. 48 (2011), no. 3, 749-782.

[11] Degtyarev, A.: Hurwitz equivalence of braid monodromies and extremal elliptic surfaces. Proc. Lond. Math. Soc. (3) 103 (2011), 1083-1120.

[12] Degtyarev, A.: The Alexander module of a trigonal curve. II. Preprint, 2012. Available at arXiv: 1202.3896.

[13] Esnault, H.: Fibre de Milnor d'un cône sur une courbe plane singulière. Invent. Math. 68 (1982), no. 3, 477-496.

[14] Kulkarni, R.S.: An arithmetic-geometric method in the study of the subgroups of the modular group. Amer. J. Math. 113 (1991), no. 6, 1053-1133.

[15] Libgober, A.: Alexander polynomial of plane algebraic curves and cyclic multiple planes. Duke Math. J. 49 (1982), no. 4, 833-851.

[16] Libgober, A.: Alexander invariants of plane algebraic curves. In Singularities, Part 2 (Arcata, Calif., 1981), 135-143. Proc. Sympos. Pure Math. 40, Amer. Math. Soc., Providence, RI, 1983.

[17] Libgober, A.: Alexander modules of plane algebraic curves. In Low-dimensional topology (San Francisco, Calif., 1981), 231-247. Contemp. Math. 20, Amer. Math. Soc., Providence, RI, 1983.

[18] Libgober, A.: Invariants of plane algebraic curves via representations of the braid groups. Invent. Math. 95 (1989), no. 1, 25-30.

[19] Libgober, A.: Problems in topology of the complements to planesingular curves. In Singularities in geometry and topology, 370-387. World Sci. Publ., Hackensack, NJ, 2007.

[20] Loeser, F. And VaquiÉ, M.: Le polynôme d'Alexander d'une courbe plane projective. Topology 29 (1990), no. 2, 163-173.

[21] OkA, M.: A survey on Alexander polynomials of plane curves. In Singularités Franco-Japonaises, 209-232. Sémin. Congr. 10, Soc. Math. France, Paris, 2005.

[22] van Kampen, E. R.: On the fundamental group of an algebraic curve. Amer. J. Math. 55 (1933), 255-267.

[23] ZaRiski, O.: On the problem of existence of algebraic functions of two variables possessing a given branch curve. Amer. J. Math. 51 (1929), no. 2, 305-328.

Received February 13, 2012; revised July 16, 2012.

Alex Degtyarev: Department of Mathematics, Bilkent University, 06800 Ankara, Turkey.

E-mail: degt@fen.bilkent.edu.tr 